ARTICLE

https://doi.org/10.1038/s41467-019-11904-4

\title{
Developmental cell death regulates lineage-related interneuron-oligodendroglia functional clusters and oligodendrocyte homeostasis
}

\author{
David Orduz (1) 1,2,5,6, Najate Benamer 1,2,3,6, Domiziana Ortolani 2,3,6, Eva Coppola2,3,4, Lisa Vigier 2,3,4, \\ Alessandra Pierani ${ }^{2,3,4}$ \& María Cecilia Angulo (1) 1,2,3
}

The first wave of oligodendrocyte precursor cells (firstOPCs) and most GABAergic interneurons share common embryonic origins. Cortical firstOPCs are thought to be replaced by other OPC populations shortly after birth, maintaining a consistent OPC density and making postnatal interactions between firstOPCs and ontogenetically-related interneurons unlikely. Challenging these ideas, we show that a cortical firstOPC subpopulation survives and forms functional cell clusters with lineage-related interneurons. Favored by a common embryonic origin, these clusters display unexpected preferential synaptic connectivity and are anatomically maintained after firstOPCs differentiate into myelinating oligodendrocytes. While the concomitant rescue of interneurons and firstOPCs committed to die causes an exacerbated neuronal inhibition, it abolishes interneuron-firstOPC high synaptic connectivity. Further, the number of other oligodendroglia populations increases through a non-cell-autonomous mechanism, impacting myelination. These findings demonstrate unprecedented roles of interneuron and firstOPC apoptosis in regulating lineage-related cell interactions and the homeostatic oligodendroglia density.

\footnotetext{
${ }^{1}$ Neurophysiology and New Microscopies laboratory, INSERM U1128, 75006 Paris, France. ${ }^{2}$ Université de Paris, 75006 and 75013 Paris, France. ${ }^{3}$ Institute of Psychiatry and Neuroscience of Paris (IPNP), INSERM U1266, 75014 Paris, France. ${ }^{4}$ Imagine Institute of Genetic Diseases, 75015 Paris, France. ${ }^{5}$ Present address: Gfi informatique, Saint-Ouen-sur-Seine, France. ${ }^{6}$ These authors contributed equally: David Orduz, Najate Benamer, Domiziana Ortolani.

Correspondence and requests for materials should be addressed to M.C.A. (email: maria-cecilia.angulo@parisdescartes.fr)
} 
D uring development, oligodendrocyte precursor cells (OPCs), the obligate progenitors of myelinating oligodendrocytes (OLs) in the Central nervous system (CNS), arise from multiple restricted periventricular germinal regions. Three sequential waves of OPCs populate the cerebral cortex according to a ventro-dorsal temporal progression ${ }^{1}$. A first wave arises from Nkx2.1-expressing precursors of the medial ganglionic eminence (MGE) and the embryonic preoptic area (ePOA) around the embryonic day 12.5 (E12.5). A second wave is generated by E14.5 from precursors expressing the homeobox gene Gsx2 in the lateral and medial ganglionic eminences (LGE and MGE) and, finally, a third wave arises at birth from precursors expressing the homeobox gene Emxl in the cortex ${ }^{1}$.

Cre-loxP fate mapping in transgenic mice revealed that the first wave of OPCs (firstOPCs) from the MGE and ePOA is eliminated 10 days after birth in the mouse cerebral cortex, and replaced by OPCs produced in the second and third waves ${ }^{1}$. Although firstOPCs survive in other CNS regions ${ }^{1,2}$, their massive death in the neocortex makes unlikely any role of this OPC population in cortical circuit maturation and myelination ${ }^{1,3}$. Furthermore, the genetic ablation of firstOPCs led to the conclusion that this cell population may play redundant functions with other OPCs since they are replaced with no drastic alterations of myelination ${ }^{1,2}$. In fact, OPCs tend to homeostatically maintain their cell number ${ }^{4,5}$, and it is assumed that competition among OPC waves participates to this process during development ${ }^{1}$.

As firstOPCs, the majority of cortical GABAergic interneurons are born from progenitors expressing the transcription factor $\mathrm{Nkx} 2.1$ settled in the MGE and $\mathrm{ePOA}^{6}$. While the MGE produces around $60 \%$ of the entire interneuron population, the ePOA contributes with $\sim 10 \%$ (refs. ${ }^{6-8}$ ). Beyond this common embryonic origin, interneurons and OPCs are close partners during cortical development. First, migrating interneurons signal to OPCs in a paracrine fashion by secreting factors that promote oligodendrogenesis ${ }^{9,10}$. Furthermore, OPCs represent the only non-neuronal cell type in the CNS that receive functional synaptic inputs from neurons and are innervated by GABAergic interneurons ${ }^{11-13}$. In the mouse somatosensory cortex, the GABAergic synaptic activity of OPCs reaches a peak at postnatal day 10 (PN10) and then declines ${ }^{12,14,15}$. Restricted to a precise temporal window, OPC connectivity is characterized by a highly sophisticated spatial organization of interneuron-OPC microcircuits ${ }^{15}$. Interestingly, this transient connectivity occurs during the period of massive programmed cell death of both cortical interneurons and $\mathrm{OPCs}^{1,12,15,16}$. Indeed, the entire firstOPC population ${ }^{1}$ and $40 \%$ of interneurons ${ }^{16}$ are eliminated during the first two postnatal weeks. The convergence in the embryonic origin of interneurons and firstOPC, their death and their highly regulated transient interneuron-OPC connectivity suggests possible interactions between these two cell types that might participate to cortical construction.

Challenging the above-mentioned established views, our results demonstrate that not all firstOPCs die in the developing neocortex and that the surviving subpopulation displays a specific spatial distribution and a preferential synaptic connectivity with their ontogenetically-related interneurons. Furthermore, the induction of the concomitant survival of MGE- and ePOAderived interneurons and firstOPCs committed to die causes a significant decrease of interneuron-firstOPC connection probability while generating an exacerbated neuronal inhibition. Therefore, although rescued interneurons are functional and target other neurons, they lack their preferential connectivity with firstOPCs. Finally, the prevention of interneuron and firstOPC apoptosis causes a general increase in the entire oligodendroglia population and a hypermyelination of deep cortical layers after the end of the period of massive cortical programmed cell death. Our results contradict the notion that different OPC waves play redundant roles and compensate for each other.

\section{Results}

Connectivity between Dbx1-derived interneurons and firstOPCs. The common embryonic origin of interneurons and firstOPCs may impact the transient and very local arrangement of interneuron-OPC microcircuits previously reported in the somatosensory cortex ${ }^{15}$. Progenitors expressing the transcription factor Dbx1 in the ePOA produce a subset of firstOPCs that begin the invasion of cortical territories by E14 (refs. ${ }^{17,18}$ ). In addition, they also give rise to a small, but very diverse population of interneurons that preferentially invades deep cortical layers ${ }^{7,8,17}$. To specifically follow firstOPCs and interneurons from the ePOA at postnatal stages, we produced $D b \times 1^{C R E} ; R_{0 s a 26^{Y F P} ; N G 2^{D s R e d}}$ triple transgenic mice. In this mouse line, interneurons and firstOPCs derived from Dbx1-expressing progenitors of the ePOA were lineage-traced with the fluorescent reporter YFP, and OPCs from all origins with DsRed ${ }^{18,19}$. We initially examined sections of the somatosensory cortex at PN10 when interneurons reach a peak of synaptic connectivity with OPCs ${ }^{15}$. As expected from previous reports $7,8,17, \mathrm{YFP}^{+}$cells were scarce and distributed mainly in cortical layers V and VI (Fig. 1a). Interestingly, we observed that instead of appearing homogeneously distributed, a majority of them were rather prone to gather together by forming small cell groups spatially segregated from one another (Fig. 1a). To assess the presence of firstOPCs in these groups, we searched for $\mathrm{YFP}^{+} / \mathrm{DsRed}^{+}$cells and verified their identity by co-labeling with the oligodendroglial lineage marker Olig2 (Fig. 1a). Groups of Dbx1-derived cells were composed of $\mathrm{YFP}^{+}$interneurons only, $\mathrm{YFP}^{+} / \mathrm{DsRed}^{+}$OPCs only or $\mathrm{YFP}^{+}$interneurons and $\mathrm{YFP}^{+} /$ DsRed $^{+}$OPCs simultaneously. This narrow spatial arrangement of $\mathrm{YFP}^{+} / \mathrm{DsRed}^{+}$OPCs with their ontogenetically related interneurons suggests potential specific interactions between these two cell types.

OPCs synaptically interact with interneurons by forming very local microcircuits during the second postnatal week ${ }^{15}$. We thus compared the synaptic connection probability of presynaptic $\mathrm{YFP}^{+}$interneurons with either $\mathrm{YFP}^{+} / \mathrm{DsRed}^{+}$OPCs (from Dbx1-expressing progenitors) or $\mathrm{YFP}^{-} / \mathrm{DsRed}^{+}$OPCs (from other sources) using paired recordings in layers $\mathrm{V}$ and $\mathrm{VI}$ of acute somatosensory cortical slices during the second postnatal week. Patched cell pairs never exceeded intersomatic distances of $80 \mu \mathrm{m}$ to remain in the spatial range of interneuron-OPC connections ${ }^{15}$. We found that the stimulation of presynaptic $\mathrm{YFP}^{+}$interneurons elicited synaptic currents on neighbor $\mathrm{YFP}^{+} / \mathrm{DsRed}^{+}$OPCs in $42.8 \%$ of tested pairs (Fig. 1b, c). The evoked postsynaptic currents (PSCs) were completely abolished by the $\mathrm{GABA}_{\mathrm{A}}$ receptor antagonist SR95531 (Gabazine), confirming the GABAergic nature of these synapses (Fig. 1b). Interestingly, the connection probability was significantly reduced when considering all interneuron-OPC tested pairs (23.8\%) or pairs with $\mathrm{YFP}^{-} /$ DsRed $^{+}$OPCs (13.5\%, Fig. 1c). The 3.2-fold higher connection probability of postsynaptic $\mathrm{YFP}^{+} / \mathrm{DsRed}^{+}$OPCs compared to YFP $^{-} /$DsRed $^{+}$OPCs was not accompanied by changes either in PSC amplitudes or short-term synaptic plasticity (mean amplitudes: $-5.54 \pm 1.23 \mathrm{pA}$ vs. $-5.57 \pm 1.26 \mathrm{pA}$, respectively, $p=0.98$ paired-pulse ratio: $0.45 \pm 0.03$ vs. $0.49 \pm 0.06$, respectively, $p=$ 0.55 , Mann-Whitney $U$ test; data represent mean \pm SEM). In addition, we observed a peak of connectivity at PN10-11 for both $\mathrm{YFP}^{+} / \mathrm{DsRed}^{+}$OPCs and $\mathrm{YFP}^{-} / \mathrm{DsRed}^{+}$OPCs (Fig. 1d), indicating that the connectivity of $\mathrm{YFP}^{+}$interneurons with OPCs derived from distinct origins followed the similar developmental regulation of the entire interneuron population ${ }^{15}$. 

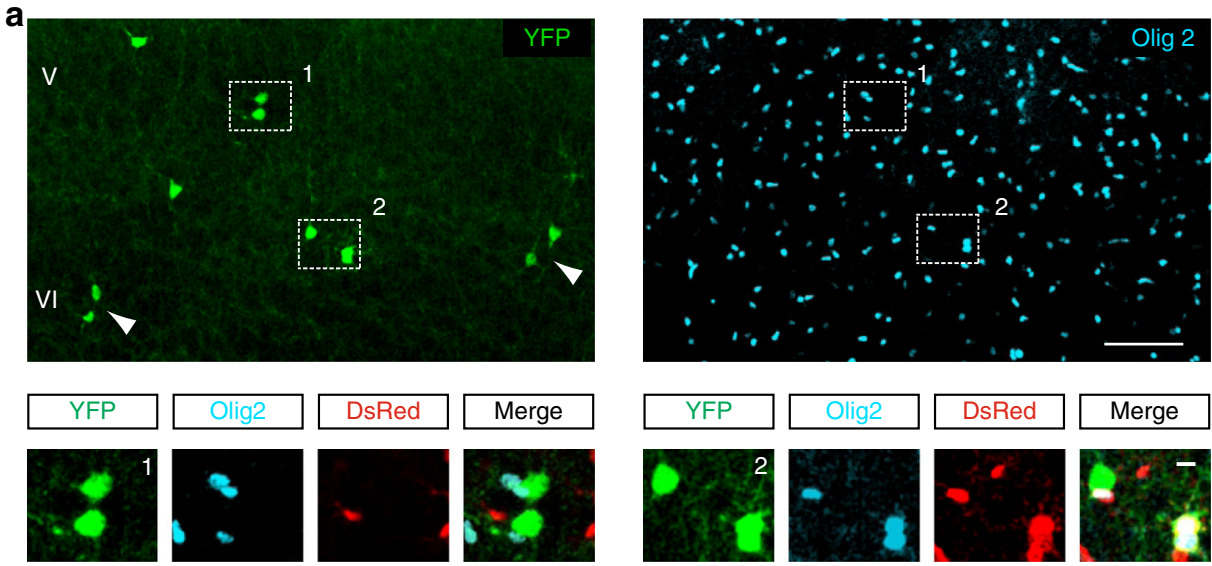

b

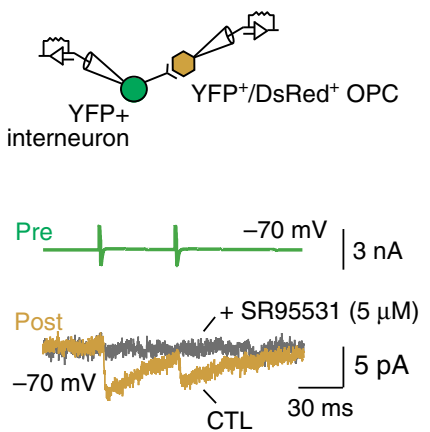

e

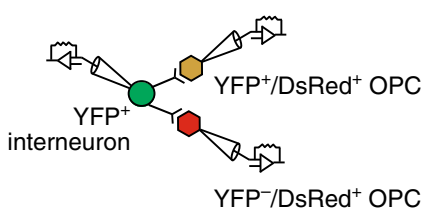

C
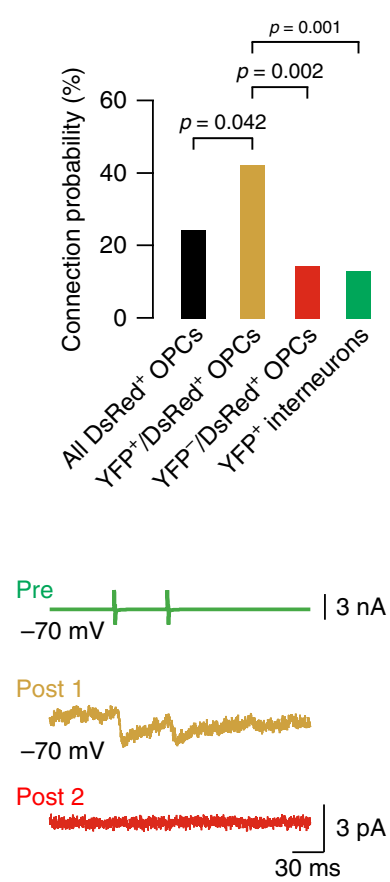

d

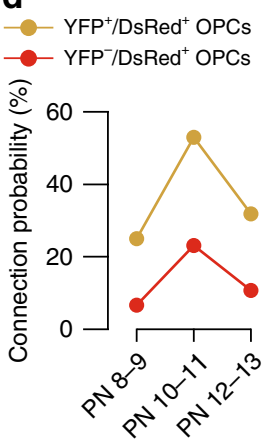

f

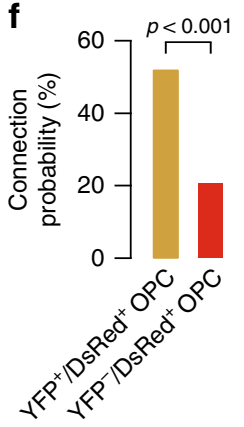

Fig. 1 Dbx1-derived interneurons preferentially target OPCs from the same lineage. a Confocal images of YFP+ interneurons (green) and YFP $+/ D s R e d+$

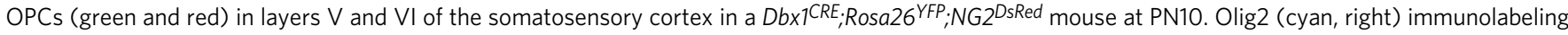
for the same cortical field identifies oligodendroglia within these groups. White dotted squares surround two YFP+ cell groups shown in insets. The first group (1) is composed of two YFP+ interneurons and the second (2) of a YFP+ interneuron and two YFP $+/ \mathrm{DsRed}^{+} / \mathrm{Olig}^{+} \mathrm{OPCs}^{+}$. Arrowheads point to two other groups of YFP+ interneurons. Scale bars: 100 and $10 \mu \mathrm{m}$. b Paired recording between a presynaptic YFP+ interneuron and a YFP + /DsRed ${ }^{+}$OPC. $^{+}$ Action currents evoked in a YFP ${ }^{+}$interneuron (green) elicited PSCs recorded in a YFP ${ }^{+} / D_{\text {sRed }}+$ OPC (yellow; average of 100 traces) that were abolished by the GABA $\mathrm{A}_{\mathrm{A}}$ receptor antagonist SR95531 ( $5 \mu \mathrm{M}$, gray; $n=5$ connected pairs). c Connection probabilities for all tested DsRed ${ }^{+}$OPCs $($black, $n=168)$, $\mathrm{YFP}^{+} /$DsRed + OPCs (yellow, $n=56$ ), YFP-/DsRed + OPCs (red, $n=118$ ) and postsynaptic YFP+ interneurons (green, $n=72$ ). Note that presynaptic $\mathrm{YFP}^{+}$interneurons target preferentially $\mathrm{YFP}^{+} /$DsRed $^{+}$OPCs compared to YFP-/DsRed ${ }^{+}$OPCs or postsynaptic YFP ${ }^{+}$interneurons (Chi-squared test; significant $p$-values are indicated). d Connection probabilities for YFP $+/ D_{\text {RRed }}+$ OPCs (yellow) and YFP-/DsRed + OPCs (red) as a function of three postnatal stages (PN8-9, PN10-11 and PN12-13; $n=12, n=17, n=6$ tested pairs for YFP + /DsRed + OPCs and $n=15, n=26, n=17$ tested pairs for YFP - / DsRed ${ }^{+}$OPCs). e Sequential paired recordings between a single presynaptic YFP + interneuron (green) and two different neighbor OPCs, a YFP ${ }^{+} / \mathrm{DsRed}^{+}$ OPC (yellow) and a YFP ${ }^{-}$/DsRed+ OPC (red). Note that action currents evoked in the YFP+ interneuron elicited PSCs in the YFP+/DsRed + OPC, but not in the YFP-/DsRed + OPC. $f$ Connection probabilities for sequential paired recordings revealed a high connection probability for YFP $+/ D^{2}$ Red + OPCs (yellow) compared to $\mathrm{YFP}^{-} / \mathrm{DsRed}^{+}$OPCs (red; $n=58$ sequential paired recordings) (Chi-squared test)

The preference of $\mathrm{YFP}^{+}$interneurons to innervate $\mathrm{YFP}^{+} / \mathrm{DsRed}^{+}$ OPCs suggests that interneuron-OPC connectivity is positively influenced by the embryonic origin. However, this preferential connectivity could also result from a higher capacity of $\mathrm{YFP}^{+}$ interneurons to innervate any surrounding cell when organized in $\mathrm{YFP}^{+}$cell groups. Since $\mathrm{YFP}^{+}$interneurons were also often close to each other (Fig. 1a), we tested their synaptic connectivity when their intersomatic distances were $<80 \mu \mathrm{m}$. Despite sharing a common 
origin, pairs of $\mathrm{YFP}^{+}$interneurons had a lower connection probability $(13.9 \%)$ than that of their ontogenetically related $\mathrm{YFP}^{+}$/ DsRed $^{+}$OPCs in the second postnatal week (Fig. 1c; Supplementary Fig. 1). In addition, we used sequential paired recordings between a single presynaptic $\mathrm{YFP}^{+}$interneuron and two distinct neighbor OPCs to compare, within the same $\mathrm{YFP}^{+}$cell group, the connection probability between $\mathrm{YFP}^{+} / \mathrm{DsRed}^{+} \mathrm{OPCs}$ and $\mathrm{YFP}^{-} / \mathrm{DsRed}^{+} \mathrm{OPCs}$ (Fig. 1e). We also observed a 2.6-fold increased connectivity onto $\mathrm{YFP}^{+} / \mathrm{DsRed}^{+}$OPCs compared to $\mathrm{YFP}^{-} / \mathrm{DsRed}^{+}$OPCs inside $\mathrm{YFP}^{+}$cell groups (Fig. 1e, f). Therefore, in comparison to other neighbor postsynaptic $\mathrm{YFP}^{+}$interneurons or OPCs from different origins, $\mathrm{YFP}^{+} / \mathrm{DsRed}^{+}$OPCs constituted the preferential synaptic target of $\mathrm{YFP}^{+}$interneurons when these two $\mathrm{YFP}^{+}$cell types were spatially associated. As for the entire populations of interneurons and $\mathrm{OPCs}^{15}$, Dbx1-derived $\mathrm{YFP}^{+}$fast-spiking interneurons (FSI) and non-fast interneurons (NFSI) innervated $\mathrm{YFP}^{+} / \mathrm{DsRed}^{+}$ OPCs and $\mathrm{YFP}^{-} / \mathrm{DsRed}^{+}$OPCs (Supplementary Fig. 2). However, $\mathrm{YFP}^{+}$FSIs constituted a prevalent presynaptic input onto any OPC (Supplementary Fig. 2c). We concluded that, in the second postnatal week, the connectivity between interneurons and firstOPCs is favored by their embryonic origin.

Dbx1-derived interneurons and firstOPCs form cell clusters. Our functional data showed a preferential connectivity between interneurons and firstOPCs derived from the ePOA in groups of $\mathrm{YFP}^{+}$cells at postnatal stages. Therefore, these cells should form predictable $\mathrm{YFP}^{+}$cell clusters throughout layers IV and VI in $D b \times 1^{C R E}$;Rosa26 ${ }^{Y F P}$ mice at PN10, i.e. at the peak of their synaptic connectivity (Fig. 1d). To test this possibility, we used unsupervised hierarchical cluster analysis via multi-scale bootstrap resampling to evaluate the existence of unbiased $\mathrm{YFP}^{+}$cell groups clustered according to their intersomatic cell distances ${ }^{20,21}$. To discriminate $\mathrm{YFP}^{+}$interneurons from $\mathrm{YFP}^{+}$ oligodendroglia in large field of views, we performed immunolabelings against YFP, the marker for the oligodendrocyte lineage Olig2 and the marker for mature OLs CC1 (Fig. 2a, b). We considered $\mathrm{YFP}^{+} / \mathrm{Olig} 2^{-} / \mathrm{CC}^{-}$cells as interneurons, $\mathrm{YFP}^{+} /$ Olig2 ${ }^{+} / \mathrm{CC}^{-}{ }^{-}$cells as firstOPCs, $\mathrm{YFP}^{+} / \mathrm{Olig} 2^{+} / \mathrm{CC}^{+}$cells as differentiated OLs and calculated Manhattan distances from their $x, y, z$ coordinates (see Methods). Figure 2c illustrates a dendrogram showing the hierarchical relationship between identified $\mathrm{YFP}^{+}$cells, grouped according to their Manhattan distances. By randomly resampling elements of the data, the bootstrap-based approach computed $p$-values for each $\mathrm{YFP}^{+}$cell cluster at each branch of the dendrogram ${ }^{20}$. Only cell groups with a $95 \%$ confidence probability were considered as clusters supported by data (Fig. 2b, c, gray boxes). This statistical analysis revealed that most $\mathrm{YFP}^{+}$cells were organized in cell clusters (Fig. 2c). We found that $72.5 \%$ of detected clusters were formed by two to three cells and 93.6\% of clusters contained a maximum of seven cells, indicating that a cluster size is restricted to few cells (Fig. 2d). We recognized clusters composed by interneurons only (62.4\%), interneurons and oligodendroglia (mixed, 26.1\%) or oligodendroglia only (Olig2 ${ }^{+}$cells, $11.5 \%$ ) (Fig. $\left.2 \mathrm{~b}, \mathrm{c}, \mathrm{e}\right)$. When considering all intersomatic distances (Euclidean) among $\mathrm{YFP}^{+}$cells in clusters, the mean distance in mixed and Olig2 + cell clusters was significantly reduced with respect to that of clusters formed exclusively by $\mathrm{YFP}^{+}$interneurons (Supplementary Fig. 3a). However, when considering clusters containing only two $\mathrm{YFP}^{+}$cells, both $\mathrm{YFP}^{+}$ interneuron and mixed clusters displayed similar distances (Supplementary Fig. 3b). The mean intersomatic distance of mixed clusters containing only two cells was limited to $70 \pm 4 \mu \mathrm{m}$ (Supplementary Fig. 3b). In line with the existence of predictable clusters, all connected pairs found with patch-clamp recordings occupied a very confined space with interneuron-OPC intersomatic distances $<60 \mu \mathrm{m}$, independently of the origin of cells (Supplementary Fig. 4).

The computation analysis and distribution of OPCs in connected pairs showed that the organization of $\mathrm{YFP}^{+}$cells in cortical layers IV-VI is not random and follows a distribution where $\mathrm{YFP}^{+}$interneurons and $\mathrm{YFP}^{+}$oligodendroglia appear often close to each other. These results indicate that the preferential connectivity of interneurons and firstOPCs from the Dbxl cell lineage can be predicted by the existence of these clusters.

Surviving Dbx1-derived OPCs produce OLs in cell clusters. It is considered that the first wave of OPCs does not play a role at postnatal stages in the neocortex because it totally disappears at around PN10 (refs. ${ }^{1,3}$ ), when the myelination process has not started yet. However, our data revealed the presence of functional lineage-related interneuron-firstOPC connections during the second postnatal week, indicating that a subpopulation of cortical firstOPCs survives. We therefore analyzed the distribution and fate of Dbx1-derived oligodendroglia during development in the somatosensory cortex (Fig. 3). We observed a seven-fold decrease in the density of $\mathrm{YFP}^{+} /$Olig2 ${ }^{+}$cells from PN4 to PN10 which were mainly firstOPCs at these ages (Fig. 3a, b). As expected, similar dynamics were observed for the total number of Olig2 ${ }^{+}$ cells and OPCs, but the decrease from PN4 to PN10 was much less pronounced (Fig. 3e, f). Together, these results indicate that firstOPCs constituted a main population of Olig2 ${ }^{+}$progenitors dying during the first postnatal days. From PN10 to PN19, the densities of $\mathrm{YFP}^{+} / \mathrm{Olig} 2^{+}$cells and all Olig2 ${ }^{+}$cells remained relatively stable (Fig. 3a, e). As for the whole population of OPCs and OLs (Fig. 3f, g), a reduction in the density of $\mathrm{YFP}^{+} / \mathrm{Olig}^{+} /$ $\mathrm{CC}^{-}$OPCs at PN10 was followed by an increase in the density of $\mathrm{YFP}^{+} / \mathrm{Olig} 2^{+} / \mathrm{CC1}^{+}$OLs at PN19 (Fig. 3b-d), indicating that surviving $\mathrm{YFP}^{+} / \mathrm{Olig} 2^{+} / \mathrm{CC}^{-}$OPCs became mature OLs (referred as firstOLs). At PN90, the density of $\mathrm{YFP}^{+} / \mathrm{Olig}^{+}{ }^{+}$cells were not significantly different to those at PN10 and PN19 but showed a tendency to decrease (Fig. 3a-c). In fact, the entire Olig ${ }^{+}$cell population also decreased at this age, probably because the brain reached its adult size (Fig. 3e). Interestingly, unsupervised hierarchical cluster analysis revealed that a majority of $\mathrm{YFP}^{+}$cells were still organized in cell clusters at PN19 and followed similar distributions and compositions to clusters at PN10 (Supplementary Fig. 3c-i). In particular, the mean intersomatic distance in mixed clusters formed by two cells was similar between the two developmental stages $(\sim 70 \mu \mathrm{m}$; Supplementary Fig. 3b, d). Finally, these clusters were also visualized in the motor and visual cortex where the dynamics of $\mathrm{YFP}^{+}$oligodendroglia followed similar trends during postnatal development (Supplementary Fig. 5).

In conclusion, $\mathrm{YFP}^{+}$interneurons and $\mathrm{YFP}^{+}$OLs keep their organization in cell clusters at later development stages, suggesting that interneurons and firstOPCs from the Dbx1 cell lineage form a functional unit that persists in the postnatal neocortex.

Dbx1-derived OLs myelinate multiple axons inside clusters. To determine the capacity of $\mathrm{YFP}^{+}$OLs to myelinate axonal fibers in the third postnatal week, we generated a $D b \times 1^{C R E}$;Rosa26 ${ }^{Y F P}$; $P L P D s R e d$ triple transgenic mice in which the proteolipid protein (PLP) promoter targets the expression of DsRed specifically in $\mathrm{CCl}^{+} \mathrm{OLs}^{4,22}$. This mouse line allowed us to lineage-trace firstOLs derived from Dbx1-expressing progenitors of the ePOA with the fluorescent reporter YFP, and OLs from all origins with DsRed. We imaged cell clusters composed of $\mathrm{YFP}^{+} / \mathrm{DsRed}^{+} \mathrm{OLs}$ and $\mathrm{YFP}^{+} / \mathrm{DsRed}^{-}$cells recognized as interneurons by their large somata (Fig. 4a, c) and, in some cases, by the expression of Parvalbumin (PV), a specific marker for FSI (Fig. 4d). Similar to 
a

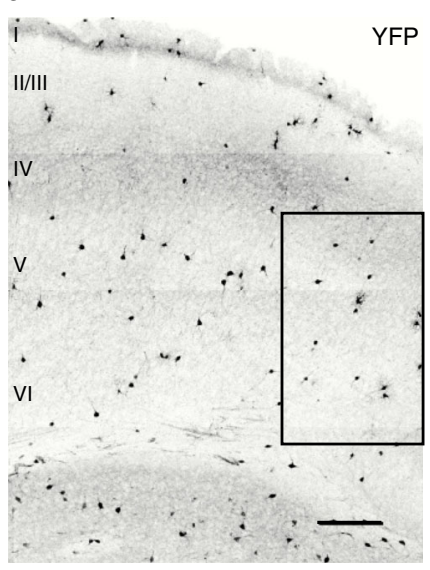

b

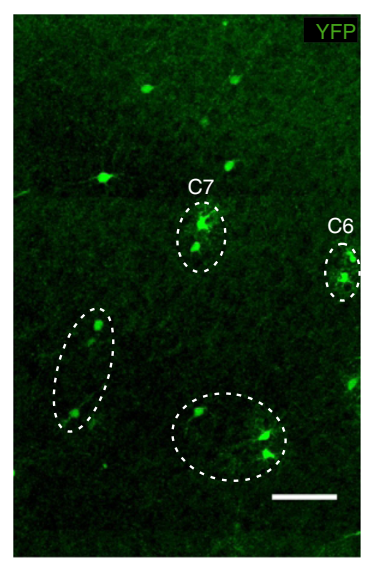

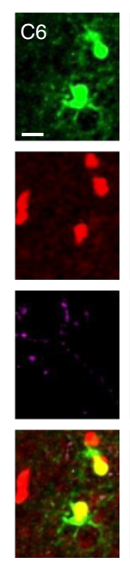

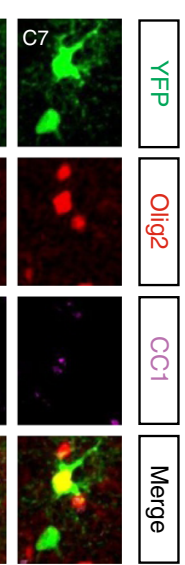

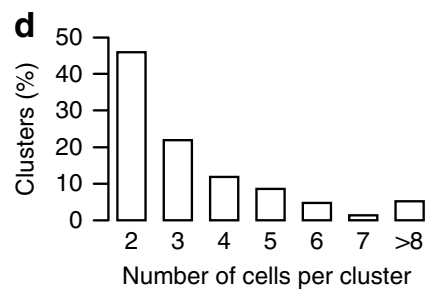

e

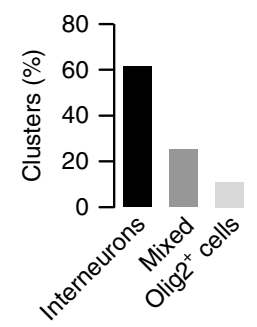

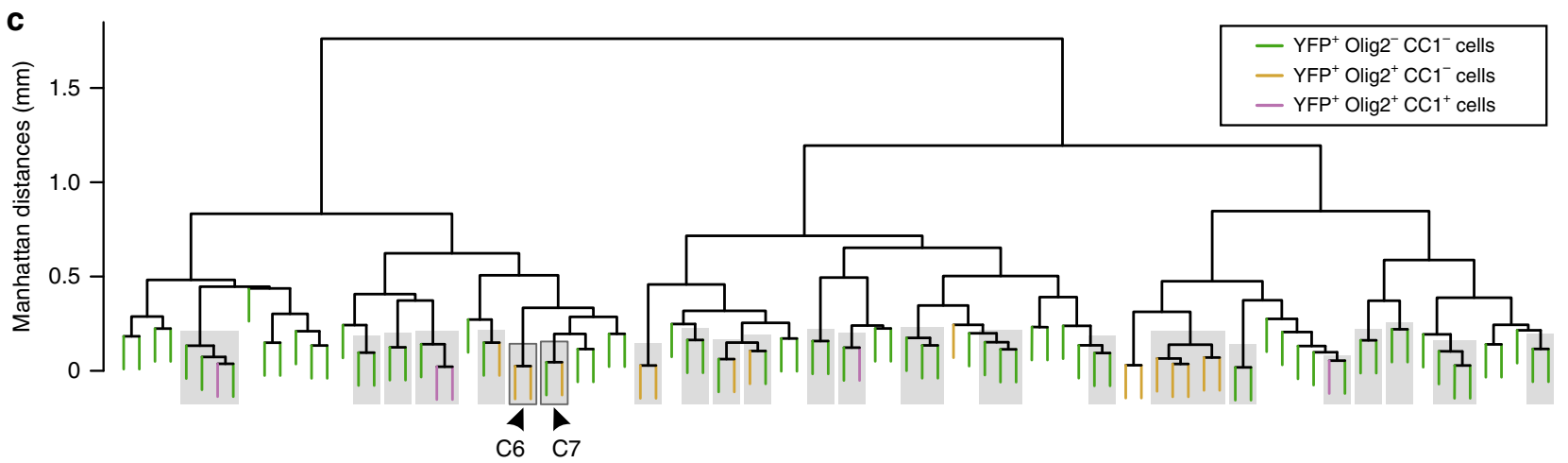

Fig. 2 Dbx1-derived interneurons and OPCs form cell clusters at PN10. a Confocal image of a coronal section of the somatosensory cortex from a PN10 Dbx ${ }^{C R E} ;$ Rosa26 YFP mouse showing YFP + cells. Scale bar: $200 \mu \mathrm{m}$. b Magnification of the rectangle in a showing the unsupervised clusters of YFP + cells detected by hierarchical cluster analysis in this region (dotted ellipses). Insets: two identified clusters containing OPCs and detected with an approximately unbiased $p$-value $\geq 0.95$. They are indicated by arrowheads in the dendrogram in $\mathbf{c}$. Note that $\mathrm{C} 6$ is formed by two $\mathrm{YFP}^{+} / \mathrm{Olig}^{+} / \mathrm{CC} 1^{-} \mathrm{OPCs}$ and $\mathrm{C7}$ by a $\mathrm{YFP}^{+}$interneuron (negative for both Olig2 and $\mathrm{CC} 1$ ) and a YFP $+/ \mathrm{Olig}^{+} / \mathrm{CC1}^{-} \mathrm{OPC}$, showing the co-existence of different cell clusters. Mature $\mathrm{YFP}^{+} /$ Olig2 ${ }^{+} / \mathrm{CC1}^{+}$OLs are rarely observed at this age. Scale bars: 80 and $10 \mu \mathrm{m}$. c Hierarchical clustering dendrogram displaying the relationship between Dbx1-derived interneurons (green), OPCs (yellow) and OLs (magenta) according to their Manhattan distances in the same slice. Detected YFP + cell clusters with approximately unbiased $p$ values $\geq 95 \%$ are shown in gray boxes while isolated cells are outside these boxes ( $72 \pm 3 \%$ cells in clusters vs. $28 \pm 3 \%$ isolated cells; $n=11$ slices from 4 mice, $p<0.0001$, Mann-Whitney $U$ test). d, e Percentages of clusters according to the number of cells per cluster (d) and the cell composition (e)

$\mathrm{PN}_{10}, \mathrm{YFP}^{+} / \mathrm{DsRed}^{+} \mathrm{OLs}$ at PN19 appeared confined around $\mathrm{YFP}^{+}$interneurons compared to $\mathrm{YFP}^{-} / \mathrm{DsRed}^{+} \mathrm{OLs}$, confirming the existence of mixed clusters formed by lineage-related interneurons and firstOLs later in development (Fig. 4e, f).

First, we performed triple immunostainings against YFP, the axonal marker SMI-312 and the myelin basic protein (MBP) (Fig. $4 \mathrm{a}, \mathrm{b}$ ). In 11 analyzed clusters, a large number of branches of $\mathrm{YFP}^{+} / \mathrm{DsRed}^{+}$OLs co-localized with both SMI-312 and MBP (Fig. 4b). Hence, differentiated $\mathrm{YFP}^{+} / \mathrm{DsRed}^{+}$OLs myelinate axons surrounding their $\mathrm{YFP}^{+}$interneuron partners (Fig. 4c). Interestingly, $\mathrm{PV}^{+}$interneurons which constitute a major input of OPCs at $\mathrm{PN} 10^{15}$ are the main myelinated GABAergic interneuron subtype in the cortex ${ }^{23-25}$. We thus tested whether $\mathrm{YFP}^{+}$/ DsRed ${ }^{+}$OLs myelinate surrounding axons of $\mathrm{PV}^{+}$interneurons. Immunostainings against SMI-312 and PV revealed that $\mathrm{PV}^{+}$ axonal segments co-localized with branches of $\mathrm{YFP}^{+} / \mathrm{DsRed}^{+}$ OLs in cell clusters (Fig. 4c, d). Quantifications showed that about a fifth of these branches were SMI- $312^{+} / \mathrm{PV}^{+}$while the others were SMI-312 $+/ \mathrm{PV}^{-}$(Fig. 4d, g). Similar proportions were observed for $\mathrm{YFP}^{-} / \mathrm{DsRed}^{+} \mathrm{OLs}$ derived from other origins outside $\mathrm{YFP}^{+}$cell clusters (Fig. 4g). Therefore, $\mathrm{YFP}^{+} / \mathrm{DsRed}^{+}$ OLs from the ePOA behave as other OLs and myelinate $\mathrm{PV}^{+}$and
$\mathrm{PV}^{-}$neurons without a preference for $\mathrm{PV}^{+}$axons, indicating that the function of these clusters is not to preferentially myelinate $\mathrm{YFP}^{+}$interneuron partners. As for the entire interneuron population $^{24,25}, 5$ out of $5 \mathrm{YFP}^{+}$FSI were myelinated while 3 out of $4 \mathrm{YFP}^{+}$NFSI were not (Supplementary Fig. 6). Finally, the branches of these $\mathrm{YFP}^{+} / \mathrm{DsRed}^{+}$OLs covered relatively small areas (Fig. 4b, d), suggesting that they myelinate GABAergic $\left(\mathrm{PV}^{+}\right)$and glutamatergic $\left(\mathrm{PV}^{-}\right)$fibers being part of the $\mathrm{YFP}^{+}$ interneuron microcircuit.

Connectivity between Nkx2.1-derived interneurons and firstOPCs. To test whether the preferential interneuron-firstOPC connectivity does not occur exclusively in the subset of Dbx1derived firstOPCs, we generated $N k x 2.1^{\text {CRE }}$;Rosa $26^{Y F P} ; N G 2^{D s R e d}$ and $N k \times 2.1^{C R E} ;$ Rosa26 $6^{\text {tdTomato }}$ transgenic mice to label all cells derived from the MGE and ePOA with YFP or tdTomato. In the first line, OPCs from other origins also expressed the DsRed. Given the large number of $\mathrm{Nkx} 2.1$-derived interneurons in these mice (around $70 \%)^{6}$, it was impossible to recognize a specific spatial organization in cell clusters (Fig. 5a). However, Nkx2.1derived OPCs and OLs followed similar dynamics to those of Dbx1-derived oligodendroglia (Fig. 5b-d). While the total 
a

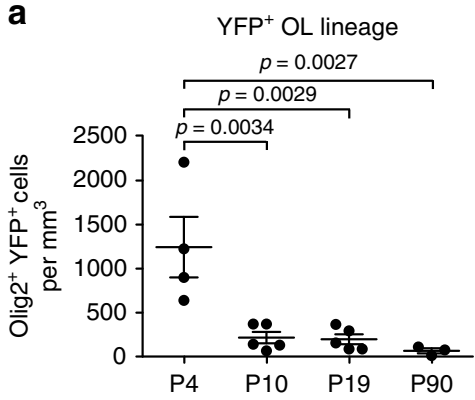

e

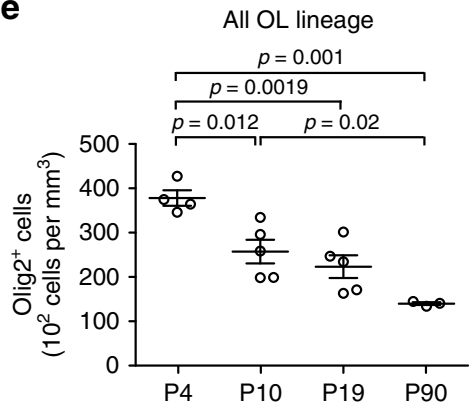

b

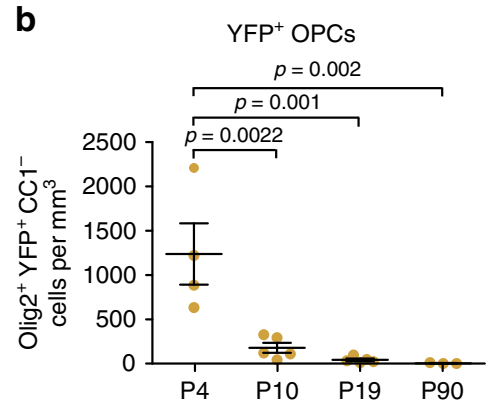

$\mathbf{f}$

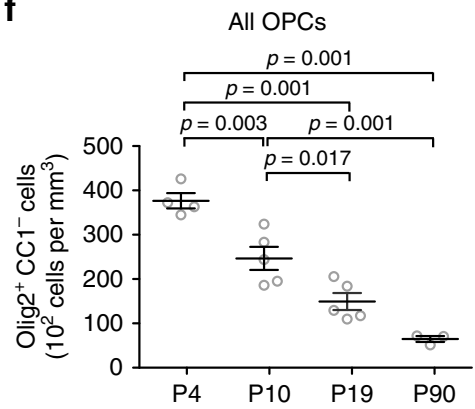

C

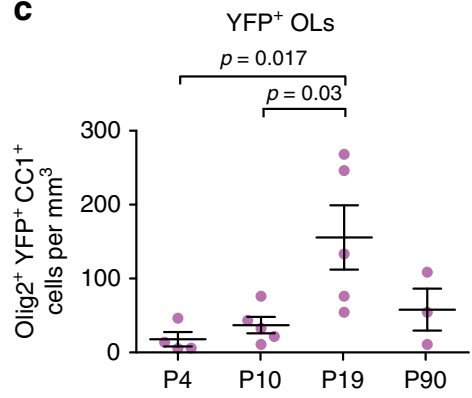

g

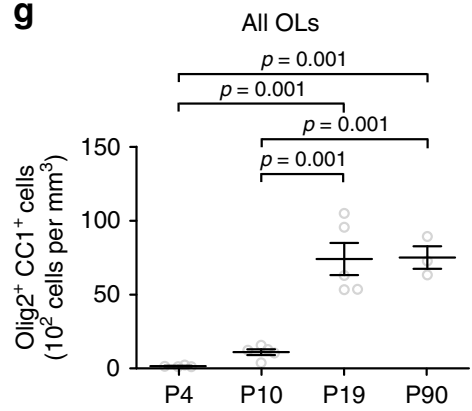

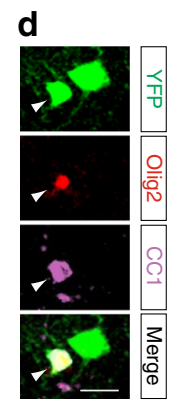

Fig. 3 Surviving Dbx1-derived OPCs become differentiated OLs. a-c Densities of YFP $+/ \mathrm{Olig}^{+}$cells (a), YFP $+/ \mathrm{Olig}^{+} / \mathrm{CC}^{-} \mathrm{OPCs}(\mathbf{b})$ and $\mathrm{YFP}^{+} / \mathrm{Olig}^{+} /$ $\mathrm{CC}^{+}$OLs (c) at PN4, PN10, PN19 and PN90 in the somatosensory cortex of Dbx 7 CRE; Rosa26 YFP mice (dots represent $n=3$ to 5 animals per age). Note that $\mathrm{YFP}^{+} / \mathrm{Olig}^{+}$cell density highly decreases from PN4 to PN10 (a). A reduction of $\mathrm{YFP}^{+} / \mathrm{Olig}^{+} / \mathrm{CC}^{-} \mathrm{OPCs}^{(\mathbf{b})}$ is followed by an increase in YFP ${ }^{+} / \mathrm{Olig}^{+} /$ $\mathrm{CC}^{+}$OLs (c) during development (one-way ANOVA test followed by a Tukey's Multiple Comparison test). $\mathbf{d}$ Confocal image of differentiated firstOLs at PN19 identified by the expression of YFP (green), Olig2 (red) and CC1 (magenta). Note the presence of a YFP+/Olig2-/CC1-interneuron in close vicinity of this $\mathrm{YFP}^{+} / \mathrm{Olig} 2^{+} / \mathrm{CC1}^{+}$OLs (white arrowhead). Scale bar: $20 \mu \mathrm{m}$. e-g Densities of total Olig2 ${ }^{+}$cells (e), Olig2 ${ }^{+} / \mathrm{CC} 1^{-} \mathrm{OPCs}_{(\mathbf{f})}$ and Olig2 ${ }^{+} / \mathrm{CC} 1^{+} \mathrm{OLs}^{-}$ (g) at PN4, PN10, PN19 and PN90 in the somatosensory cortex of Dbx $7^{C R E} ;$ Rosa26 YFP mice (dots represent $n=3$ to 5 animals per age). Note that Olig2 ${ }^{+}$ cell density decreases more from PN4 to PN10 than at later stages (e). A reduction of Olig2 $+/ C C 1^{-}$OPCs (f) is followed by an increase in Olig2 ${ }^{+} / \mathrm{CC} 1^{+}$ OLs (g) during development (one-way ANOVA test followed by a Tukey's Multiple Comparison test). Data are presented as mean \pm SEM

population of Olig2 ${ }^{+}$cells from the MGE/ePOA remained relatively stable, the OPC density decreased and OL density increased from PN10 to PN19. Then, we performed paired recordings between a fluorescent interneuron and a nearest OPC, whether from the same origin or from a different origin, during the second postnatal week (Fig. 5e, f). As for Dbx1-derived cells, Nkx2.1derived interneurons displayed a high connection probability of $38.9 \%$ with their lineage-related firstOPCs while only of $8.3 \%$ with OPCs from distinct origins (Fig. 5h). These data show that all surviving firstOPCs from both $\mathrm{MGE}$ and $\mathrm{ePOA}$ remains functionally associated with interneurons sharing a common origin in the postnatal neocortex.

Connectivity of lineage-related cells rescued from death. Large populations of interneurons and firstOPCs are eliminated by programmed cell death in the cerebral cortex during the two first postnatal weeks ${ }^{1,16}$. We thus analyzed what would be their connectivity if cells committed to die survived. To examine the impact of the concomitant rescue of lineage-related interneurons and firstOPCs on interneuron-firstOPC connectivity and neuronal circuit function, we prevented their death by the conditional deletion of the pro-apoptotic Bcl2-associated X protein (Bax) which regulates interneuron apoptosis ${ }^{16}$ and promotes the elimination of pre-oligodendrocytes ${ }^{26}$. We inactivated Bax function by crossing a mouse harboring floxed Bax alleles with $N k \times 2.1^{C R E} ;$ Rosa2 $6^{t d T o m a t o}$ mice (hereafter referred as Baxf/f mice). It is noteworthy that $D b \times 1^{C R E}$ mice could not be used in these experiments since $D b \times 1$ and Bax alleles are on the same chromosome.

An efficient rescue of $\mathrm{Nkx} 2.1$-derived interneurons, firstOPCs and firstOLs occurred in Baxf/f mice in all cortical layers at PN10 and PN19 (Supplementary Fig. 7; interneurons, 35.1\% and 32.3\% increase, firstOPCs, $59.5 \%$ and $75.1 \%$ increase and firstOLs, $61.1 \%$ and $50.5 \%$ increase at PN10 and PN19, respectively). Despite these large cell density increases, paired recordings between tdTomato ${ }^{+}$ interneurons and tdTomato ${ }^{+}$OPCs revealed a significant reduction of their connection probability from $38.9 \%$ in controls to $14.3 \%$ in Bax f/f mice during the second postnatal week (Fig. $5 \mathrm{~g}, \mathrm{~h}$ ). To corroborate this decrease in the connectivity, we analyzed GABAergic PSCs of tdTomato ${ }^{+}$OPCs evoked by extracellular stimulation in the presence of glutamate receptor antagonists at intensities known to activate a large number of fibers at this age ${ }^{23}$. Evoked GABAergic PSCs were robustly decreased in tdTomato ${ }^{+}$ OPCs of Baxf/f mice, confirming the reduced connectivity of rescued firstOPCs with interneurons (Fig. 5i, j). This low connectivity could result either from specific disruption of lineage-related interneuron-firstOPC interactions or a lack of capacity of rescued interneurons to form synapses and integrate neuronal networks. To distinguish between these possibilities, we used acute thalamocortical slices to analyze neuronal excitatory and inhibitory PSCs in a simple circuit motif where layer IV glutamatergic neurons receive a weak excitatory thalamic input compared to FSI which activation triggers powerful intracortical feedforward inhibition ${ }^{27}$ (Fig. 6a). As expected in control $^{27}$, electrical thalamic stimulation induces direct small excitatory PSCs and large disynaptic inhibitory PSCs in layer IV glutamatergic neurons in the third postnatal week (Fig. 6a, b). Therefore, unlike a defect of rescued interneurons to form synapses, neuronal inhibitory currents were robustly increased in Baxf/f mice, resulting in a strong decrease in the excitation/inhibition ratio (Fig. 6b, c). Furthermore, while 4 out of 8 paired recordings between FSI and glutamatergic neurons were connected in control, 7 out of 7 were 
a
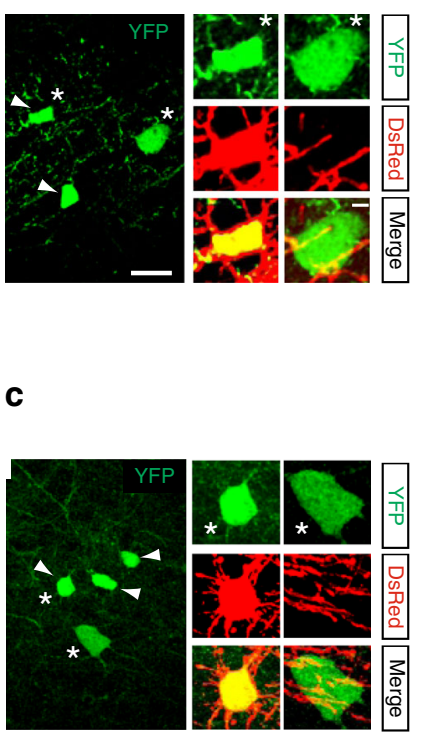

b
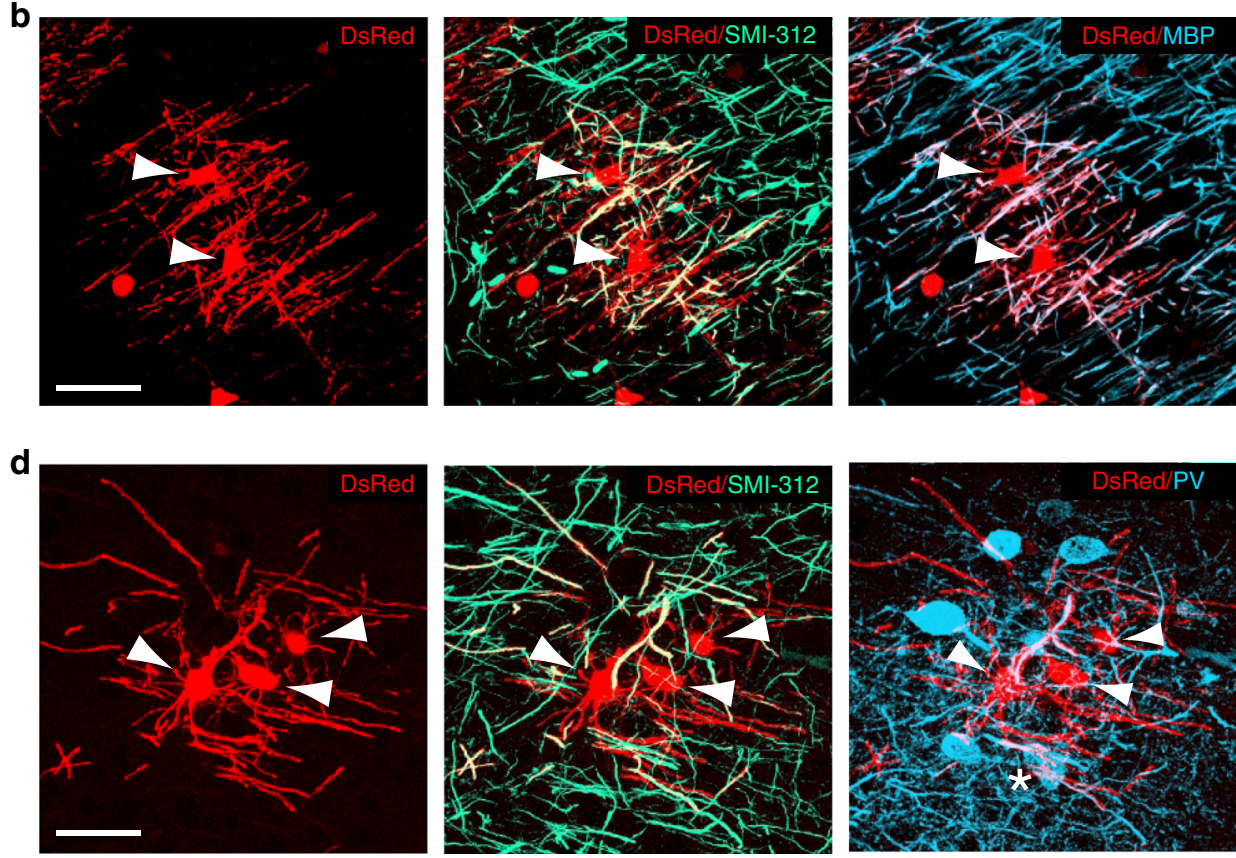

g $\circ \triangle \mathrm{YFP}^{+}$DsRed $\mathrm{d}^{+} \mathrm{OLs}$ $\circ \triangle$ YFP $^{-}$DsRed ${ }^{+}$OLs
YFP' DsRed $^{+}$OLs

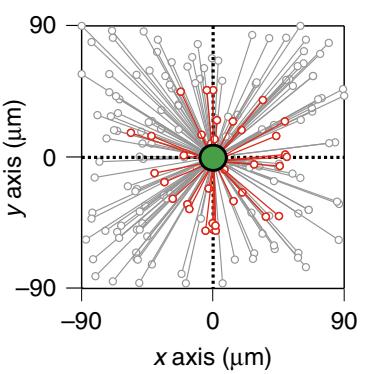

f

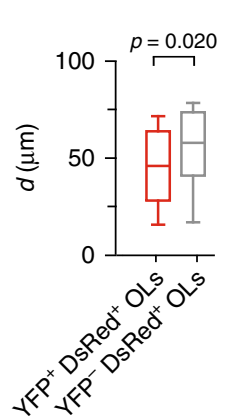

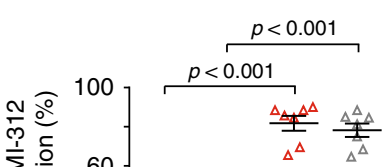

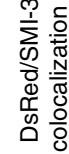
$20 \_$

$\frac{\frac{\circ}{\frac{0_{0}^{\circ}}{0}}}{P V}$

$$
\mathrm{PV}^{+} \quad \mathrm{PV}^{-}
$$

Fig. 4 Dbx1-derived OLs myelinate axons of $\mathrm{PV}^{+}$and $\mathrm{PV}^{-}$neurons in cell clusters. a, c Confocal images of two YFP + cell clusters composed of YFP + / DsRed $^{+}$OLs (white arrowheads) and YFP + interneurons at PN19 in the somatosensory cortex of Dbx 7 CRE; Rosa26 ${ }^{Y F P}$;PLPDsRed mice. Insets: magnification of a YFP+/DsRed + OL and a YFP+ interneuron of the same clusters (asterisks). Scale bars: 25 and $5 \mu \mathrm{m}$. $\mathbf{b}$ The DsRed + branches of OLs belonging to the cluster in a (top, white arrowheads) co-localize with the axonal marker SMI-312 (middle) and MBP (bottom), confirming that these cells are myelinating cells ( $n=24$ firstOLs from $n=3$ mice). d The DsRed ${ }^{+}$branches of OLs belonging to the cluster in $\mathbf{c}$ (top, white arrowheads) co-localize with the axonal marker SMI-312 (middle) and PV, the specific marker of FSI (bottom), confirming that some branches myelinate $\mathrm{PV}+$ interneurons. The asterisk indicates the soma of the PV+/YFP+ interneuron in the cluster (mean area occupied by YFP+/DsRed + OL: $13419 \pm 698 \mu m^{2} ;$ mean diameter: $155.57 \pm 7.88 \mu$ m; $n=$ 9 from 9 clusters). Scale bars in $\mathbf{b}$ and $\mathbf{d}: 50 \mu \mathrm{m}$. e Spatial distribution of YFP ${ }^{+} /$DsRed $^{+}$OLs (red) and YFP $^{-} /$DsRed $^{+} \mathrm{OLs}$ (gray) with respect to YFP ${ }^{+}$ interneurons (green) in YFP+ cell clusters $(n=22$ clusters). Note the shorter intersomatic distances for OLs and interneurons from the Dbx1 lineage, but the lack of a specific orientation of OLs. $\mathbf{f}$ Box plots of distances (d) of YFP+/DsRed + OLs (red) and YFP-/DsRed ${ }^{+}$OLs (gray) to YFP+ interneurons (mean intersomatic distance: $44.2 \pm 3.5 \mu \mathrm{m}$ for $n=33 \mathrm{YFP}^{+} / \mathrm{DsRed}^{+}$OLs compared to $53.5 \pm 2.1 \mu \mathrm{m}$ for $n=99 \mathrm{YFP}^{-} / \mathrm{DsRed}^{+}$OLs, respectively, in 22 clusters; Mann-Whitney $U$ test; significant $p$-value is indicated). Boxes show interquartile ranges and medians; whiskers indicate $10 \%$ and $90 \%$ percentile values. g Dot plots of percentages of $\mathrm{PV}^{+}$and $\mathrm{PV}^{-}$axons co-localizing with SMI-312 and DsRed + branches of YFP $+/ D^{+}$Red + OLs (red; $n=7$ clusters from 3 mice) and YFP-/DsRed ${ }^{+}$OLs (gray; $n=7$ clusters from 3 mice) (two-way ANOVA, $F=0.978$ and DF $=1$; significant $p$-values are indicated). Data in $\mathbf{g}$ are presented as mean $\pm \mathrm{SEM}$

connected in Baxf/f mice (Fig. 6d, e). Connected pairs in this mouse line displayed larger inhibitory PSCs than controls without changing their short-term synaptic plasticity and thus their presynaptic release properties (Fig. 6d-f). This suggests that rescued interneurons, in addition to be supernumerary, formed more synaptic contacts into their postsynaptic glutamatergic neurons than controls. Hence, the exceeding number of interneurons in Bax f/f mice caused a hyper-innervation of other neurons, enhancing the inhibitory drive of neuronal networks and leading to a drastic excitation/inhibition imbalance. In contrast, they lost their preferential innervation with their lineage-related firstOPCs. Nkx2.1-derived interneurons rescued from death were thus functional and contacted other neurons, but their survival did not promote interactions with firstOPCs.

These findings show that the connectivity between interneurons and firstOPCs sharing a common origin constitute a highly specific and regulated process that, unlike for neurons, cannot be promoted by increasing cell densities. Since forcing the survival of 


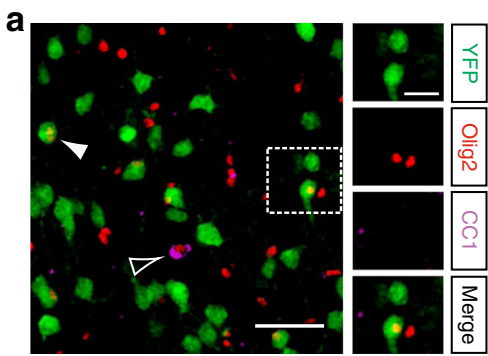

e

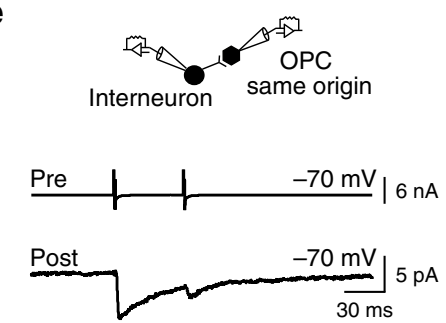

h

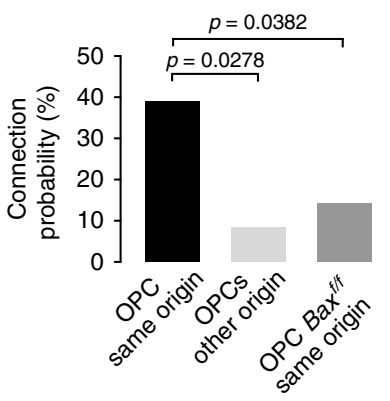

b

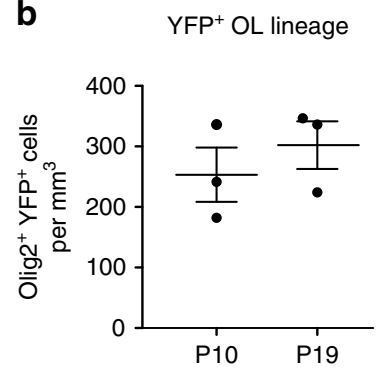

f
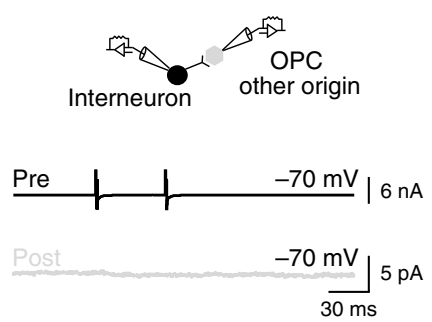

i

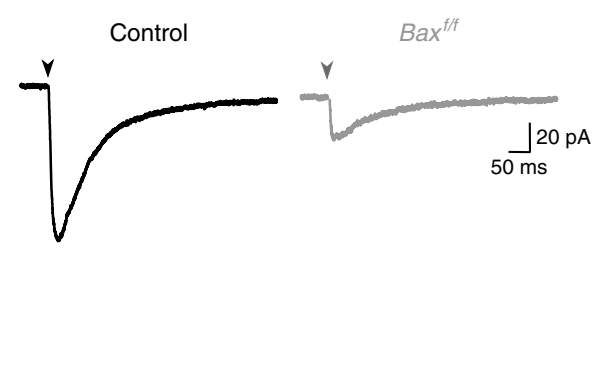

C

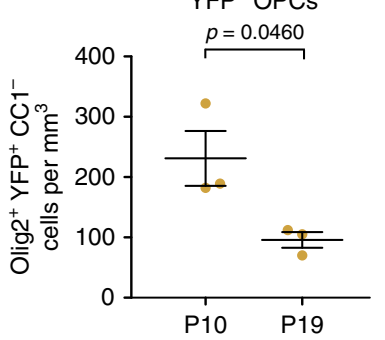

d

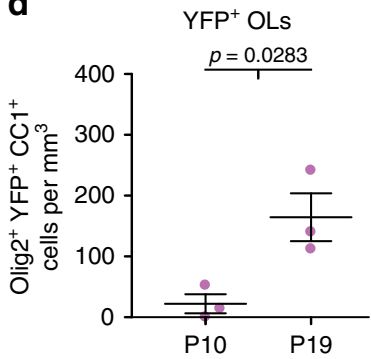

$\mathbf{g}$
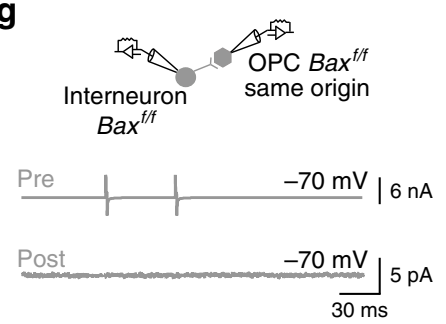

j

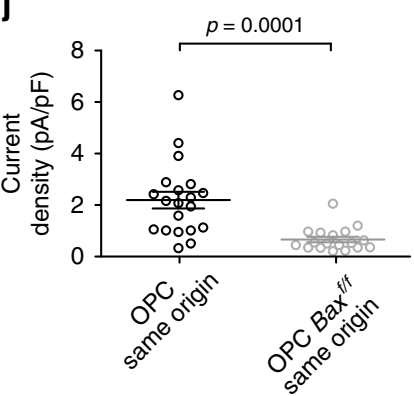

Fig. 5 The preferential interneuron-firstOPC connectivity is reduced in Baxt/f mice. a Confocal images of YFP+ (green), Olig2 ${ }^{+}$(red) and CC1 ${ }^{+}$cells in layer $\checkmark$ of the somatosensory cortex in a Nkx2.1 ${ }^{C R E} ; R_{\text {Rosa2 }} 6^{Y F P}$ mouse at PN10. The white dotted square surrounds a YFP + interneuron and a YFP $+/ O$ lig2 ${ }^{+} / \mathrm{CC}^{-}{ }^{-}$ OPC shown in insets. Another YFP ${ }^{+} / \mathrm{Olig}^{+} / \mathrm{CC1}^{-} \mathrm{OPC}$ (solid arrowhead) and a YFP $-/ \mathrm{Olig} 2^{+} / \mathrm{CC}^{+} \mathrm{OL}$ (open arrowhead) are indicated. Scale bars: 100 and $20 \mu \mathrm{m}$. b-d Layer $\mathrm{V}$ and $\mathrm{VI}$ densities of YFP $+/ \mathrm{Olig} 2^{+}$cells $(\mathbf{b})$, YFP $+/ O$ lig2 ${ }^{+} / \mathrm{CC1}^{-} \mathrm{OPCs}(\mathbf{c})$ and $\mathrm{YFP}^{+} / \mathrm{Olig}^{+} / \mathrm{CC1}^{+} \mathrm{OLs}^{(\mathbf{d})}$ at PN10 and PN19 in the somatosensory cortex of $N k \times 2.1^{C R E}$; Rosa26 YFP mice (dots represent $n=3$ mice per age). e-g Paired recordings between a presynaptic fluorescent interneuron and an OPC of the same origin (e) or a different origin (f) in control mice, or between a presynaptic tdTomato ${ }^{+}$interneuron and a tdTomato ${ }^{+}$ OPC of the same origin in Baxf/f mice ( $\mathbf{g}$ ). $\mathbf{h}$ Connection probabilities for OPCs of the same origin in control (black, $n=36$ ), OPCs of different origin in control (light gray, $n=24$ ) and OPCs of the same origin in Baxf/f mice (dark gray, $n=35$ ). Note that presynaptic interneurons target preferentially OPCs of the same origin in control (black) compared to OPCs of different origin (light gray) or OPCs of same origin but after Bax inactivation (dark gray; Chisquared test; significant $p$-values are indicated). i GABAergic PSCs evoked by stimulation of neuronal fibers (100 $\mu$ s; $20 \mathrm{~V}$ ) in firstOPCs of control (black) and $B a x f / f$ mice (dark gray) recorded at $-70 \mathrm{mV}$ in the presence of $10 \mu \mathrm{M}$ 2,3-dioxo-6-nitro-1,2,3,4-tetrahydrobenzoquinoxaline-7-sulfonamide (NBQX) and $50 \mu \mathrm{M}$ D-(-)-2-amino-5-phosphonopentanoic acid (D-AP5), showing a reduced mean GABAergic PSC in the Bax $/ / f$ mouse. Stimulation artefacts were blanked for visibility. The stimulation time is indicated (arrowheads). $\mathbf{j}$ Dot plots of current densities for firstOPCs in control (black; $n=20$ ) and Bax $f / f$ (dark gray; $n=18$ ) mice calculated by dividing the mean amplitude of evoked PSCs by the cell capacitance. Note the decreased PSC densities in knockout mice (Mann-Whitney $U$ test; significant $p$-value is indicated). Data in $\mathbf{b}-\mathbf{d}$ and $\mathbf{j}$ are presented as mean $\pm \mathrm{SEM}$

these cell types does not promote their connectivity, our data also suggest that, in the normal postnatal neocortex, the firstOPCs that display low levels of connectivity undergo apoptosis while those highly connected survive (Fig. 7f).

Programmed cell death is key for oligodendroglia homeostasis. The genetic ablation of specific OPC waves from ventral regions does not modify the total OPC number and myelination in the postnatal cortex, suggesting that competition of OPCs from different origins compensate from one another ${ }^{1}$. An imbalance in the firstOPC number should therefore be compensated by a reduction of OPCs from other sources to maintain the correct cell density. To test whether the increase in firstOPCs in Baxf/f mice is counterbalanced by a reduction in OPCs from different origins, we quantified the densities of tdTomato ${ }^{-}$OPCs and tdTomato ${ }^{-}$OLs in layers I-III, VI and V-VI. At PN10, the increased density of
tdTomato $^{+} /$Olig2 $^{+}$cells in Bax $f / f$ mice was not accompanied by a reduction of non-recombinant tdTomato ${ }^{-}$OPCs and OLs in any cortical layer (Supplementary Fig. 8). Later in development, after the end of the massive programmed cell death of interneurons and firstOPCs in the cortex ${ }^{28}$, quantifications of non-recombinant tdTomato $^{-}$oligodendroglia revealed a significant increase in the density of this glial cell population in Bax f/f mice (Fig. 7; increase of 39\% of tdTomato-/Olig2 ${ }^{+}$cells considering all layers at PN19). While tdTomato ${ }^{-}$OPCs were significantly increased in layers I-III where there is less myelin than in deep cortical layers (Fig. 7b-d; 64\% increase of tdTomato- OPCs in I-III layers), tdTomato $^{-}$OLs were highly increased in layers IV and V-VI of Bax $f / f$ mice (Fig. $7 \mathrm{~b}-\mathrm{d} ; 44 \%$ and $38 \%$ increase of tdTomato- OLs in layer IV and V-VI, respectively). Consequently, we observed a significant increase in myelination, detected by myelin basic protein (MBP), in layer V and VI at PN19 (Fig. 7d, e). These 
a

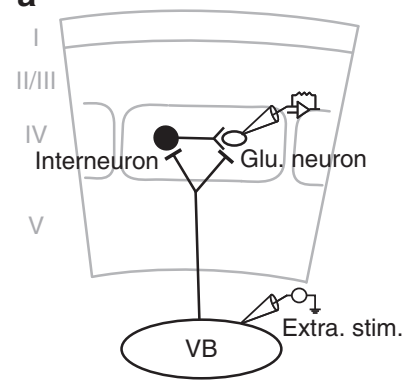

d

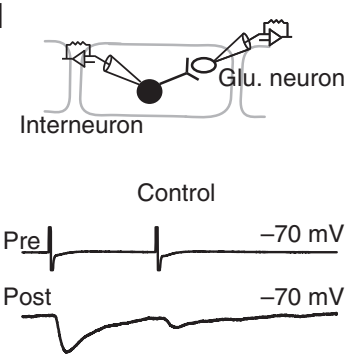

b

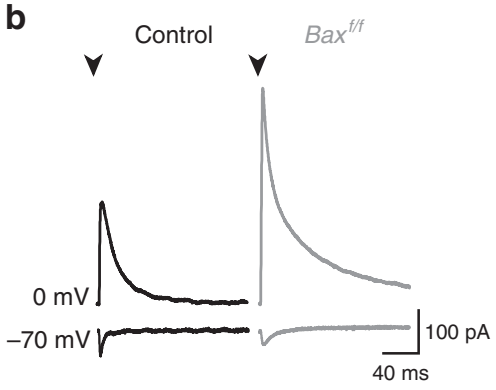

e

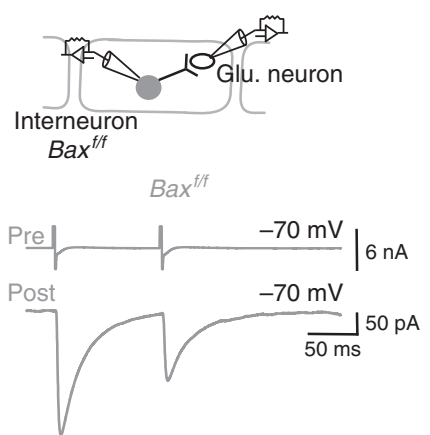

c

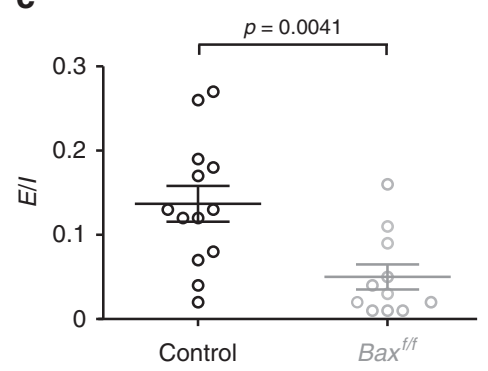

f

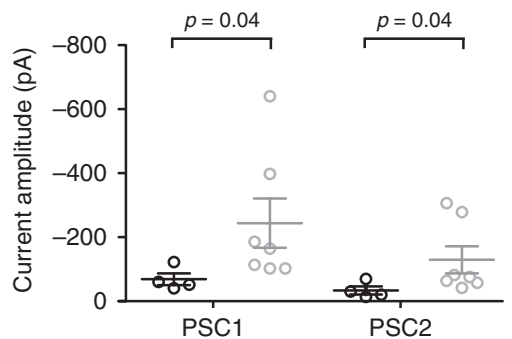

Fig. 6 An exceeding number of interneurons increases inhibition in neuronal networks. a Diagram representing the experimental procedure where a layer IV glutamatergic neuron recorded during the extracellular stimulation of the ventro-basal nucleus (VB) of the thalamus. In this circuit, the recorded neuron is synaptically connected by FSI which also receive thalamic input that triggers a strong disynaptic feedforward inhibition. b Excitatory (inward) and inhibitory (outward) PSCs evoked by thalamic stimulation in layer IV glutamatergic neurons of control (black) and Bax $/ / f$ mice (dark gray) recorded at -70 and $0 \mathrm{mV}$, respectively, in acute thalamocortical slices during the third postnatal week. Stimulation artifacts were blanked for visibility. The stimulation time is indicated (arrowheads). c Dot plots of excitation/inhibition ( $E / I)$ ratio obtained for glutamatergic neurons in control (black, $n=13$ ) and Baxf/f (dark gray, $n=10$ ) mice by dividing excitatory PSCs by inhibitory PSCs (Mann-Whitney $U$ test; significant $p$-value is indicated). $\mathbf{d}$, e Paired recordings between a presynaptic FSI and a layer IV glutamatergic neuron in control (black, d) and Bax f/f (dark gray, e) mice. Note that action currents evoked in FSI elicited larger PSCs in glutatamergic neurons of Baxf/f mice. $\mathbf{f}$ Dot plots of PSCs evoked by the first (PSC1) and second (PSC2) action current in the FSI (Mann-Whitney $U$ test; significant $p$-value are indicated). The paired-pulse ratio (PPR $=$ PSC1/PSC2) was not different between control (black, $n=4$ out of 8 pairs connected) and $B a x^{f / f}$ (dark gray, $n=7$ out of 7 pairs connected) mice, indicating that there were no changes in the release probability of presynaptic FSI (PPR: $0.45 \pm 0.05$ and $0.52 \pm 0.07$, respectively; $p=0.412$, Mann-Whitney $U$ test). Data are presented as mean \pm SEM

results indicate that the prevention of cell death of lineage-related interneurons and firstOPCs induced a major imbalance in other oligodendroglia populations that impacted myelination at late developmental stages.

Overall, these findings show that the population of rescued tdTomato ${ }^{+} / \mathrm{Olig} 2^{+}$cells was not compensated by a reduction of OPCs from other sources. On the contrary, the concomitant persistence of lineage-related interneurons and firstOPCs committed to die induced a pronounced oligodendroglia imbalance through a non-cell-autonomous mechanism, after the period of massive cortical programmed cell death (Fig. $7 \mathrm{~g}$ ). Therefore, oligodendroglia homeostasis in the developing cortex is not solely determined by competition among different OPC waves. Apoptosis is thus not only required to properly set interneuron-firstOPC interactions and reach a correct excitation/inhibition balance of neuronal networks, but also to regulate the proper densities of OPCs and myelinating OLs across different cortical layers.

\section{Discussion}

Revealing how interneurons and OPCs interact during postnatal development is fundamental for our understanding of how these different cellular subtypes contribute to the assembly, maturation and myelination of cortical circuits. Here we shed light on a complex postnatal interplay between interneurons and firstOPCs derived from the same embryonic origin, characterized by the existence of functional cell clusters that unexpectedly display a high synaptic connectivity. Late in development, surviving firstOPCs differentiate into mature oligodendrocytes inside cell clusters where they myelinate different types of neuronal fibers in vicinity of their interneuron counterparts. Therefore, the region of birth in the embryo and the cell lineage favor postnatal cellular interactions that are tightly regulated during development. Indeed, the connectivity between lineage-related interneurons and firstOPCs is not promoted by increasing their cell densities (Fig. 7f, g). Furthermore, contrary to the idea that OPC populations from distinct embryonic origins compensate with each other to homeostatically control OPC density $^{1}$, the concomitant increase of lineage-related interneurons and firstOPCs is not translated into a decrease in other OPC populations but to a high increase in the entire oligodendroglia population via a non-cell-autonomous mechanism (Fig. 7f, g). Our findings question the idea that the different waves of OPCs compete with each other to regulate OPC density and point to unprecedented roles of developmental death of interneurons and firstOPCs in regulating their lineage-related cell interactions and the homeostasis of oligodendroglia.

Our initial hypothesis was that the common origin between interneurons and firstOPCs favors the assembly of interneuronOPC microcircuits that display a very local arrangement ${ }^{15}$. Here we show that the cell lineage favors both the distribution of cells in predictable clusters and their preferential high local connectivity. Moreover, Bax-dependent rescue experiments indicate that interneurons and firstOPCs prevented from death induces a decrease rather than an increase in the proportion of lineage- 
a
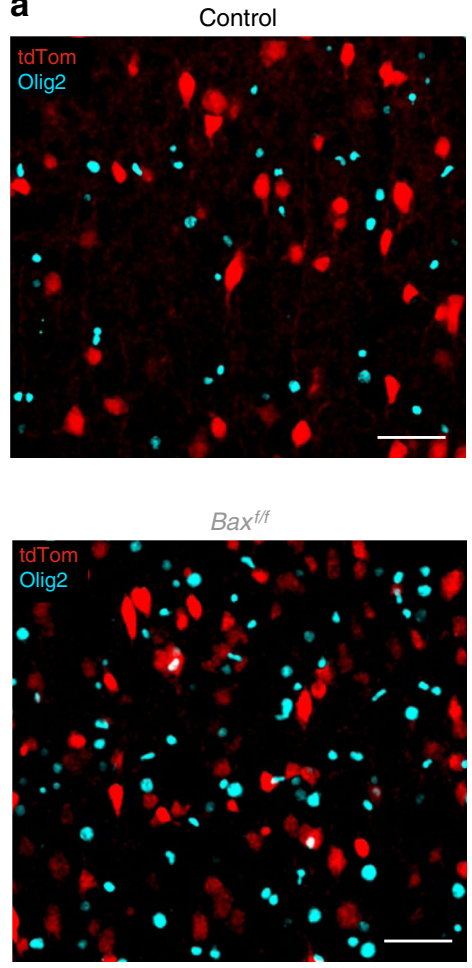

2 FirstOPCs

Interneurons from MGE and ePOA

FirstOCPs and Interneurons eliminated by PCD

TI FirstOLs

Other OPCs

Other OLs b

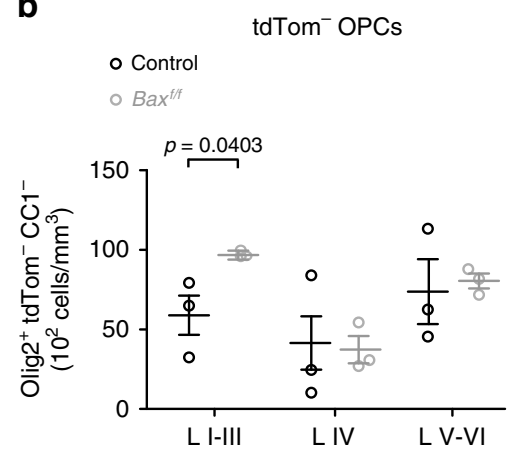

C

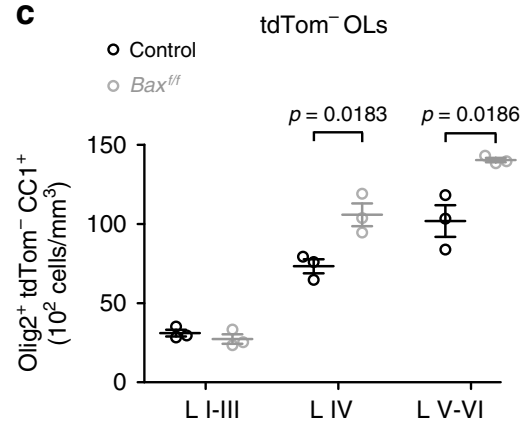

d

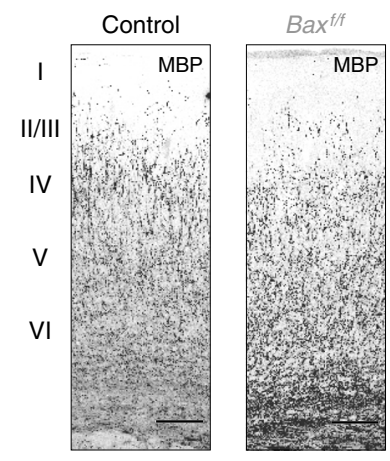

e

$$
\begin{aligned}
& \text { - Control } \\
& \text { - Bax }
\end{aligned}
$$

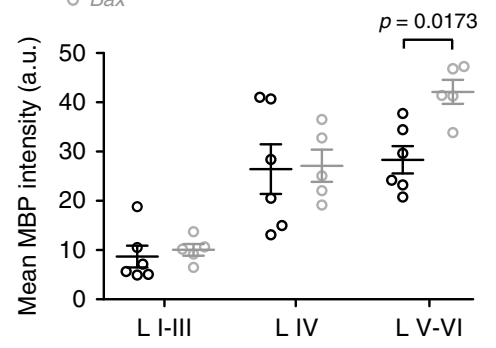

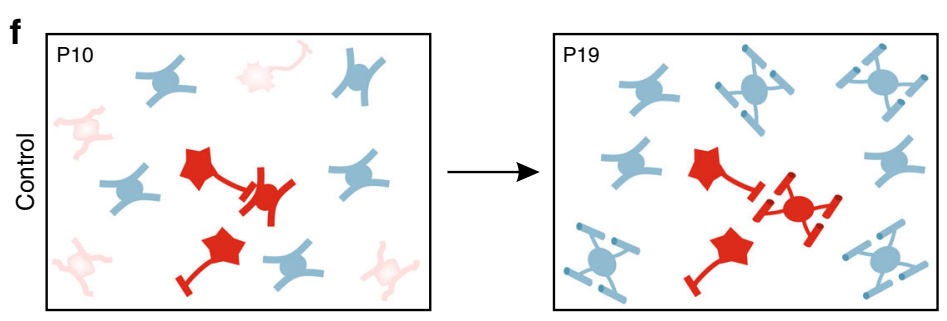

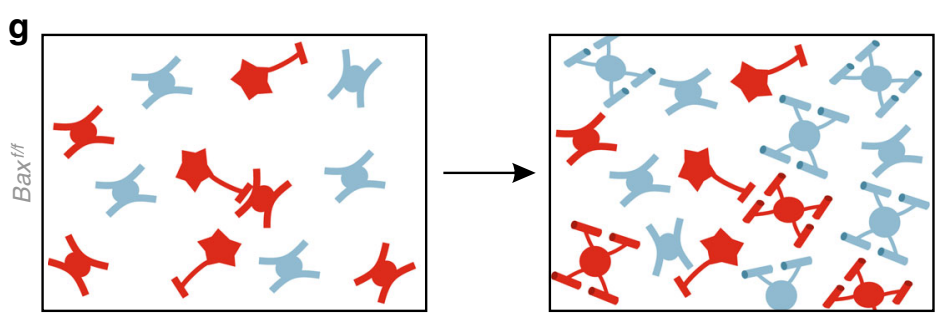

Fig. 7 Imbalance of oligodendroglia homeostasis and hypermyelination in Baxf/f mice at PN19. a Confocal images of tdTomato (red) and Olig2 ${ }^{+}$(cyan) cells of layers $\mathrm{V}$ and $\mathrm{VI}$ of the somatosensory cortex in control (left) and Bax $f / f$ (right) mice at PN19. Note the large increase in the number of nonrecombinant tdTomato ${ }^{-} / \mathrm{Olig}^{+}$oligodendroglia in Baxt/f mice. Scale bar: $50 \mu \mathrm{m}$. b, c Densities of non-recombinant tdTomato ${ }^{-} / \mathrm{Olig}^{+} / \mathrm{CC}^{-} \mathrm{OPCs}(\mathbf{b})$ and tdTomato ${ }^{-} / \mathrm{Olig}^{+} / \mathrm{CC} 1^{+}$OLs (c) in layers I-III, IV and V-VI of the somatosensory cortex in control (left) and Baxf/f (right) mice at PN19 (dots represent $n=3$ animals per condition; Mann-Whitney $U$ test; significant $p$-values are indicated). $\mathbf{d}$ Confocal images of MBP in the somatosensory cortex of control (left) and Baxt/f (right) mice at PN19. The characteristic gradient of increasing myelination from superficial to deep cortical layers occurs in both controls and Baxf/f mice. Note the increased myelination of deep layers in the Baxf/f mouse. Scale bar: $200 \mu$ m. e Dot plots of mean MBP fluorescence intensities in layers I-III, IV and V-VI of the somatosensory cortex in control ( $n=6$ slices from 2 animals) and Bax $f / f$ ( $n=5$ slices from 2 animals) mice at PN19 (Mann-Whitney $U$ test; significant $p$-value is indicated). Data are presented as mean \pm SEM. $\mathbf{f}, \mathbf{g}$ Schematic diagram illustrating postnatal functional clusters between interneurons and oligodendroglia derived from the MGE and ePOA (red), same cell types already eliminated by programmed cell death (PCD, pink) and oligodendroglia from other sources (blue) in control (f) and Bax $f / f(\mathbf{g})$ mice during postnatal development. Note the decreased connection probability of firstOPCs in Baxf/f mice with respect to controls at PN10 and the increase in both recombinant (red) and non-recombinant (blue) oligodendroglia in Baxf/f mice ( $\mathbf{g}$ ) with respect to controls (f) at PN19. A non-cell-autonomous mechanism impacts the number of non-recombinant oligodendroglia as these cells are not targeted in Bax ${ }^{f / f}$ mice at PN19

related interneuron-firstOPC synaptic connections. Since Baxdependent rescue of firstOPCs circumvents synaptic activity, our results suggest that those firstOPCs that display low levels of connectivity undergo apoptosis in normal conditions (Fig. 7f, g). This is in line with a role of these neuron-glia synapses in OPC survival $^{23}$. Interestingly, a recent report demonstrates that interneuron survival depends on their high levels of connectivity by pyramidal neurons during the period of interneuron programmed cell death ${ }^{29}$. Synaptic activity seems therefore to be a crucial factor favoring both neuron and OPC survival. The sophisticated organization in small clusters formed by interneurons and firstOPCs establishing functional interactions also 
recall previous reports describing a delicate anatomical and functional interplay between clonal pyramidal neurons in the network ${ }^{30}$. Nevertheless, reports on how cortical GABAergic interneurons are organized in the cortex remain contradictory. While interneurons labeled at the clonal level tend to distribute into local clusters of few neurons ${ }^{31,32}$, findings using barcoded retrovirus libraries for large-scale analyses of specific identified clonal cells show that interneurons disperse broadly in the neocortex ${ }^{33,34}$. A more recent report shows that spatially clustered interneurons from the MGE and ePOA obtained by low-titer retrovirus-injected radial glia in the embryo develop electrical but not chemical synapses after $\mathrm{PN} 14^{35}$. Although the non-random allocation of interneurons derived from MGE/ePOA seems acknowledged by all authors, restricted clustering of sibling interneurons is still a matter of debate. In this report, interneurons and firstOPCs inside cell clusters are unlikely to be clonal since the massive interneuron production occurs two days before that of firstOPCs: E10.5 for Nkx2.1-derived interneurons ${ }^{36}$ and E12.5 for firstOPCs ${ }^{1}$. However, this point as well as the molecular and cellular factors allowing for the joint positioning of these two cell types in deep cortical layers will need further investigation.

Recent reports show that 5-15\% of myelinated deep-layer axons belong to GABAergic $\mathrm{PV}^{+} \mathrm{FSI}^{23-25}$. In addition, the protein composition of myelin enwrapping non-GABAergic and GABAergic axons differ, the latter expressing 20\% more $\mathrm{MBP}^{24}$. Considering the recently established OL heterogeneity 37 , we could speculate that pyramidal cells and interneurons are myelinated by distinct OLs endowed with the ability to produce different myelin. However, our data show that individual OLs, derived or not from the ePOA, myelinate similar proportions of $\mathrm{PV}^{+}$and $\mathrm{PV}^{-}$axons and thus neurons of different nature and origins. Whether a single OL has the ability to produce myelin segments with distinct molecular composition according to the neuronal subtype remains unknown. Moreover, the lack of preference of firstOLs for $\mathrm{PV}^{+}$ axons in $\mathrm{YFP}^{+}$cell clusters suggests that interactions between lineage-related interneurons and firstOLs do not guide the firstOL to preferentially myelinate the axon of its interneuron partner. Thus, activity-dependent myelination by firstOLs probably does not follow a predetermined ontogenetic program. Nevertheless, firstOLs remain close to their lineage-related interneurons and their processes project towards different directions covering a whole distance of around $150 \mu \mathrm{m}$ which ensures the myelination of multiple axons in proximity of their interneuron counterparts. Therefore, the proximity of firstOLs to Dbx1-derived interneurons might substantially contribute to myelination and maturation of specific local circuits by facilitating action potential propagation and synchronization of glutamatergic and GABAergic neurons within a restricted space. In line with this, neuronal circuits formed by interneurons onto pyramidal cells are confined with a high connection probability at intersomatic distances $<200 \mu \mathrm{m}^{38}$.

Although OPCs are highly motile ${ }^{4,5}$, it is more likely that these progenitors stay in the same cluster rather than move from a cluster to another during postnatal development. In fact, cultured OPCs derived from ventral regions, which includes firstOPCs, have less inherent migration capabilities than OPCs from dorsal regions ${ }^{39}$. Furthermore, in adult demyelinating lesions, OPCs derived from ventral regions display a reduced capacity to proliferate and differentiate into mature OLs compared to those of dorsal regions ${ }^{39}$. Although RNA-sequencing did not reveal differences in the gene expression profile among OPC populations ${ }^{2}$, unappreciated functional differences of OPCs from distinct origins are emerging, underlying the importance of functional analyses of specific cell types.

Oligodendroglia are over-generated during development and subsequently eliminated by programmed cell death during the first two postnatal weeks ${ }^{1,40,41}$. It was recently showed that the transcription factor EB (TFEB) promotes the death of premyelinating OLs in a Bax-dependent manner, a mechanism controlling the spatial and temporal specificity of brain myelination ${ }^{26}$. In the neocortex, the three waves of OPCs are thought to contribute to the homeostatic regulation of these progenitors by competition ${ }^{1}$. In fact, cortical OPCs have an incredible capacity to regulate their own density throughout life. In the developing somatosensory cortex, a sensory (whisker) deprivation causes an increased death of newly-formed OLs that is accompanied by an enhanced OPC proliferation ${ }^{4}$. OPC differentiation or ablation also triggers OPC migration and proliferation in the adult, a process that rapidly restore cell density ${ }^{5}$. OPCs react to any change in the oligodendroglia population to compensate for its loss and ensure the correct myelination of neuronal circuits. Only severe pathological conditions such as chronic hypoxia in the young ${ }^{13}$ or progressive Multiple Sclerosis in the adult ${ }^{42}$ perturb the OPC capacity to preserve the homeostasis of its pool. However, a potential impact of neuron-glia interactions on oligodendroglia homeostasis had not been previously explored. Challenging previous views, our findings show that the rescue of lineage-related interneurons and firstOPCs committed to die induces an unexpected global increase in oligodendroglia density (Fig. 7f, g). These findings reveal a new role of programmed cell death during cortical development in controlling the oligodendroglia number via a non-cell-autonomous mechanism.

The mammalian cortex is unique as it is the only structure in the CNS that hosts different transient cell populations that almost completely disappear at early postnatal stages: Cajal Retzius neurons, subplate neurons, cortical plate transient neurons and firstOPCs ${ }^{28}$. In addition, glutamatergic projection neurons and GABAergic interneurons also undergo a significant cell death that induces $30-40 \%$ reduction of their initial population ${ }^{28}$. The precise functions of this previously underestimated cellular death are still unknown, but they are probably not restricted to a simple elimination of supernumerary cells. Interestingly, recent data show that pyramidal neuron apoptosis is critical to regulate interneuron survival and stabilize excitatory-inhibitory ratios of cortical networks ${ }^{29}$. Beyond these findings, our study shows that the death/survival balance of interacting neurons and glia is key to build their long-term interactions and ensures the proper myelination and construction of cortical circuits after the phase of massive cell death in the cortex.

\section{Methods}

Transgenic mice. The experiments of the present study followed European Union and institutional guidelines for the care and use of laboratory animals and were approved by the French ethical committee for animal care of the University Paris Descartes (Committee $\mathrm{N}^{\circ} \mathrm{CEEA34}$ ) and the Ministry of National Education and Research (Project No: 13094-2017081712355709). Several transgenic lines were produced. First, Cre lines driven by the Dbx1 and Nkx2.1 promoter were used as heterozygous to generate $D b \times 1^{C R E}$;Rosa $26^{Y F P}, N k \times 2.1^{C R E}$;Rosa $26^{Y F P}$ and $N k \times 2.1^{C R E}$; Rosa26 ${ }^{\text {tdTomato }}$ transgenic mice and lineage-trace cells derived from the $\mathrm{POA}^{18}$ and MGE and ePOA ${ }^{1}$. Dbx $1^{C R E} ; \operatorname{Rosa} 26^{Y F P}$ and $N k x 2.1^{C R E} ;$ Rosa $26^{Y F P}$ mice were also crossed with the NG2 ${ }^{D s R e d}$ heterozygous transgenic line which allowed us to recognize the whole OPC population by the expression of DsRed ${ }^{19}$. In another set of experiments, the $D b x 1^{C R E} ; R o s a 26^{Y F P}$ mice was bred with the $P L P^{D s R e d}$ mice $^{22}$ which allowed us to recognize the OL population by the expression of DsRed. Finally, the $B a x^{t m 2 S j k} ; B a k 1^{t m 1 T h s n / J}$ line ${ }^{43}$ harboring the floxed Bax allele and the Bak knock-out allele (stock $\mathrm{N}^{\circ} 006329$; Jackson Laboratories) was crossed with the $N k x 2.1^{C R E}$;

Rosa26 ${ }^{\text {tdTomato }}$ line to inactivate Bax and permanently label Nkx2.1-derived cells. We

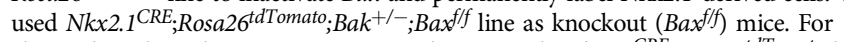
electrophysiological experiments, we used as controls $N k x 2.1^{C R E} ;$ Rosa26 $6^{\text {tdTomato }}$ line when recorded OPCs needed to be identified by fluorescence, otherwise Cre negative Nkx2.1 ${ }^{\text {CRE }}$;Rosa26 $6^{\text {tdTomato }} ;$ Bak $^{+/-} ;$Bax $1^{f / f}$ mice from the same littermates of Bax f/f mice were used. For cell countings, we used as controls $N k x 2.1^{C R E}$;Rosa26 $6^{\text {tdTomato; }}$ $\mathrm{Bak}^{+/-} ; \mathrm{Bax}{ }^{f /+}$ animals from the same littermates of Bax $f / f$ mice. Dbx1-expressing progenitors of the ePOA generate interneurons in deep cortical layers and firstOPCs in all layers; other Dbxl-derived neuronal types such as layer I Cajal Retzius neurons and cortical plate transient neurons in upper layers are not generated from the ePOA and were not considered in this study $7,8,17,18,28$. No other Dbxl-derived neuron or glial cell types have been reported in the cerebral cortex ${ }^{17}$. Animals were genotyped by PCR using primers specific for the different alleles and maintained in the animal 
facility under $12 \mathrm{~h}$ light/dark cycle with ad libitum access to food and water. Both female and male were indiscriminately used.

Acute slice preparation. Most experiments were performed using $300-\mu \mathrm{m}$-thick acute parasagittal slices of the barrel cortex from transgenic mouse with an angle of $10^{\circ}$ to the sagittal plane $\mathrm{e}^{12,15}$. In experiments aiming to analyze the $E / I$ ratio of layer IV glutamatergic neurons we performed $350-\mu \mathrm{m}$-thick tangential thalamocortical slices. An Olympus BX51 microscope equipped with a $\times 40$ fluorescent water-immersion objective allowed us to visualize YFP and DsRed fluorescent proteins by means of excitation beams supplied by Blue and Green Optoleds (Optoled Light Sources, Cairn Research, UK). Two sets of excitation/emission filters were used (470 and $525 \mathrm{~nm}$ filters for YFP, and 560 and $620 \mathrm{~nm}$ filters for DsRed) and images were collected and acquired with an iXon+ 14-bit digital camera (Andor Technology, UK) and with Imaging Workbench 6.0 software (Indec Biosystems, USA), respectively.

Paired recordings and extracellular stimulation. Electrophysiological experiments were performed at RT using an extracellular solution containing (in $\mathrm{mM}$ ): $126 \mathrm{NaCl}, 2.5 \mathrm{KCl}, 1.25 \mathrm{NaH}_{2} \mathrm{PO}_{4}, 26 \mathrm{NaHCO}_{3}, 20$ glucose, 5 pyruvate, $3 \mathrm{CaCl}_{2}$, and $1 \mathrm{MgCl}_{2}\left(95 \% \mathrm{O}_{2}, 5 \% \mathrm{CO}_{2}\right)$. During paired recordings, presynaptic interneurons were recorded with an intracellular solution containing (in $\mathrm{mM}$ ): $130 \mathrm{~K}$ gluconate (KGlu), $10 \mathrm{GABA}, 0.1 \mathrm{EGTA}, 0.5 \mathrm{CaCl}_{2}, 2 \mathrm{MgCl}_{2}, 10 \mathrm{HEPES}, 2 \mathrm{Na}_{2}-$ ATP, $0.2 \mathrm{Na}$-GTP, and $10 \mathrm{Na}_{2}$-phosphocreatine $(\mathrm{pH} \approx 7.3)$, and postsynaptic cells (OPCs, interneurons and layer IV glutamatergic neurons) with an intracellular solutions containing (in $\mathrm{mM}$ ): $130 \mathrm{CsCl}, 54$-aminopyridine, 10 tetraethylammonium chloride, 0.2 EGTA, $0.5 \mathrm{CaCl}_{2}, 2 \mathrm{MgCl}_{2}$, 10 HEPES, $2 \mathrm{Na}_{2}$-ATP, $0.2 \mathrm{Na}-\mathrm{GTP}$, and $10 \mathrm{Na}_{2}$-phosphocreatine $(\mathrm{pH} \approx 7.3)$. During extracellular stimulation, layer IV glutamatergic neurons were recorded with a similar intracellular solution but containing $125 \mathrm{mM} \mathrm{CsCH} \mathrm{SO}_{3} \mathrm{H}(\mathrm{CsMeS})$ instead of $\mathrm{CsCl}(\mathrm{pH} \approx 7.3)$. Extracellular stimulations were obtained using either a monopolar electrode (glass pipette) placed in layers V and VI near the OPC recorded with a CsCl-based intracellular solution or a bipolar concentric electrode placed in the thalamic nucleus while recording layer IV glutamatergic neurons in CsMeS-based intracellular solution $(100 \mathrm{~ms}$ pulse, 5-40 V; Iso-Stim 01D, npi electronic $\mathrm{GmbH}$, Tamm, Germany). Potentials were corrected for a junction potential of $-10 \mathrm{mV}$ when using KGlu- and CsMeS-based intracellular solution.

Whole-cell recordings were performed with a Multiclamp 700B and signals filtered at digitized at $4 \mathrm{kHz}$ and $20 \mathrm{kHz}$ respectively. Off-line analysis of digitized data was performed using pClamp10.1 software (Molecular Devices) and Neuromatic package within IGOR Pro 6.0 environment (Wavemetrics, USA) ${ }^{44}$. A paired recording was considered as connected when the average of PSCs recorded in the postsynaptic cells was 2-fold larger than the standard deviation of the noise. Paired-pulse ratios were calculated as PSC2/PSC1 ${ }^{15}$. The $E / I$ of ratio of layer IV glutamatergic neurons was calculated as EPSCs/IPSCs.

The spatial $x-y$ coordinates of connected and unconnected $\mathrm{YFP}^{+}$interneurons and OPCs were extracted from DIC images. The recorded slice was oriented to fix the $y$ axis as the shorter imaginary line from the soma of the $\mathrm{YFP}^{+}$interneuron, considered at position 0 , to the cortical surface (Supplementary Fig. 4a). The $x$ axis was parallel to the cortical surface. After obtaining the $x-y$ position of each recorded OPC with respect to the presynaptic $\mathrm{YFP}^{+}$interneuron, we calculated the intersomatic interneuron-OPC distance $(d)$, and the angle $\alpha$ with respect to the $y$ axis (Supplementary Fig. 4a).

Immunostainings and cell countings. Immunostanings were performed on perfused mice at different ages $(n=3-5 \text { animals per age })^{23}$. Animals were perfused with phosphate buffer saline (PBS) followed by $4 \%$ paraformaldehyde (PFA). Brains were postfixed during $1 \mathrm{~h}$ in PFA and stored in PBS at $4^{\circ} \mathrm{C}$. For immunostainings against Olig2, CC1, YFP and MBP, coronal vibratome slices $(100 \mu \mathrm{m})$ were prepared in PBS ice-cold solution $\left(4^{\circ} \mathrm{C}\right)$, permeabilized with $0.2 \%$ triton X100 and $4 \%$ Normal Goat Serum (NGS) for $1 \mathrm{~h}$ and incubated one night with antibodies diluted in a $0.2 \%$ triton X-100 solution and 5\% NGS. For immunostainings against PV, SMI-312 and MBP, slices were permeabilized with $1 \%$ triton X-100 and 10\% NGS overnight and incubated four nights with primary antibodies diluted in a $1 \%$ triton X-100 solution and $10 \%$ NGS. Different immunostainings were performed by using rabbit anti-Olig2 (1:400; ref. AB9610, Millipore), mouse monoclonal anti-CC1 (1:100; ref. OP80, Calbiochem), chicken anti-GFP (for detection of YFP; 1:1000; ref. A10262, ThermoFisher Scientific), rat monoclonal anti-MBP (1:100; ref. AB7349, Abcam), rabbit anti-PV (1:1000; ref. PV-27, Swant) and mouse anti-SMI-312 (1:1000; ref. 837901, Eurogentec) antibodies. All primary antibodies were washed three times in PBS and incubated in secondary antibodies coupled to Alexa-405, Alexa-488, Alexa-546 or Alexa-633 at room temperature for $2 \mathrm{~h}$ for immunostainings against Olig2, CC1, GFP and MBP (1:500) and for 2 days for immunostainings against PV, SMI-312 and MBP (1:200; Life Technologies). Confocal images were acquired using a $\times 20$ water objective or $\times 63$ oil objective with LSM-710 confocal microscope or a $\times 20$ and $\times 63$ oil objectives with a SP8 Leica confocal microscope. Images were processed and analyzed using NIH ImageJ and Imaris softwares.

For cell countings, $\mathrm{YFP}^{+}$or $\mathrm{TdTomato}^{+}$cells were identified as co-localized or not with Olig2 ${ }^{+}$and $\mathrm{CC}^{+}$(at least $n=3$ mouse per age; for each mouse, we counted $n=4$ slices). To prevent border effects in countings, cells that were at the boundaries of the analyzed volume were not considered in three of the six sides of the cube if their Olig $2^{+}$nucleus was not fully inside. To estimate the percentage of myelinated $\mathrm{PV}^{+}$and $\mathrm{PV}^{-}$axon segments per DsRed ${ }^{+} \mathrm{OLs}$, we first determine the number of DsRed ${ }^{+}$OLs branches co-localizing with SMI-312 and then determine the number of $\mathrm{PV}^{+}$and $\mathrm{PV}^{-}$branches. For MBP fluorescent intensities, images were acquired with a $\times 63$ oil objective and submitted to a background subtraction measured in layer I. Then, the mean intensity values were directly measured with NIH ImageJ using rectangle selection tool to delineate different layers.

Unsupervised cluster analysis of YFP ${ }^{+}$cells from the ePOA. The unbiased identification of $\mathrm{YFP}^{+}$cell clusters was assessed by applying an unsupervised agglomerative cluster analysis via multi-scale bootstrap resampling ${ }^{20,21}$. First, we extracted $x, y, z$ positions of layers IV-VI $\mathrm{YFP}^{+} / \mathrm{Olig}^{+} / \mathrm{CC}^{-}{ }^{-} \mathrm{OPCs}$, $\mathrm{YFP}^{+} / \mathrm{Olig} 2^{+} /$ $\mathrm{CC}^{+}$OLs and $\mathrm{YFP}^{+} / \mathrm{Olig}^{-} / \mathrm{CCl}^{-}$interneurons in the confocal sections used for cell countings at PN10 and PN19 (3-4 slices per mouse; $n=4$ mouse per age). To display the hierarchical relationship of identified $\mathrm{YFP}^{+}$cells according to their spatial proximity, we computed Manhattan intersomatic distances to produce a distance matrix and build a hierarchical dendrogram using the package hclust under the $\mathrm{R}$ environment ${ }^{21}$. It is noteworthy that Manhattan distances were used for building the dendrogram because, based in absolute distances, they are less influenced by outliers than Euclidean distances. Instead, the proximity of cells was evaluated with Euclidean distances. In the dendrogram, each identified $\mathrm{YFP}^{+}$cell is considered as a single object which repeatedly merges into higher-level clusters to its closest objects until forming a hierarchical tree. To determine whether unbiased clusters of $\mathrm{YFP}^{+}$cells were supported by data, we assessed the uncertainty of clusters at each branch of the dendrogram with the Pvclust package under the R environment ${ }^{20}$. This package computes 10,000 bootstrap samples generated by randomly resampling our experimental data and by performing bootstrap replications of the dendrogram. By computing both bootstrap probability values and approximately unbiased probability values for each cluster in the dendrogram, we inferred whether the distribution of cell clusters was random or not. If the probability of a given cluster is too low, the cluster does not exist, but if the probability is $>95 \%$, we considered that the cluster is strongly supported by the data ${ }^{20}$. Finally, once objective cell clusters were detected, we determined the number of cells per cluster, the cell composition of each cluster, and the Euclidean intersomatic distances among all cells in a cluster.

Statistics. All data are expressed as mean \pm SEM from $n$ pairs, cells or animals. GraphPad InStat software version 3.06 was used for statistical comparisons. The nonparametric two-tailed Mann-Whitney $U$ test for independent samples was used to determine statistical differences between two means. When comparisons within single pairs were required, the two-way Wilcoxon signed-rank test for related samples was used. For comparisons of cell densities, we used a one-way ANOVA test followed by a Tukey's multiple comparison post hoc test. In the case of comparisons of myelinated $\mathrm{PV}^{+}$and $\mathrm{PV}^{-}$axons by DsRed ${ }^{+}$and $\mathrm{DsReD}^{-}$OLs, we used a two-way ANOVA test followed by a multiple comparison Bonferroni's multiple comparison post hoc test. For comparisons of cluster composition and cell distances generated by hierarchical cluster analysis, each group of data was subjected to D'Agostino-Pearson normality test. Since data were not normally distributed, we used one-way Kruskal-Wallis test followed by a Dunn's multiple comparison post hoc test. Cumulative distributions were compared using Kolmogorov-Smirnov test. Correlations were tested with a Pearson $r$ test and differences were considered significant when $p<0.05$.

Reporting summary. Further information on research design is available in the Nature Research Reporting Summary linked to this article.

\section{Data availability}

The data that support the findings of this study are available from the corresponding author upon reasonable request.

Received: 28 June 2019 Accepted: 4 August 2019

Published online: 18 September 2019

\section{References}

1. Kessaris, N. et al. Competing waves of oligodendrocytes in the forebrain and postnatal elimination of an embryonic lineage. Nat. Neurosci. 9, 173-179 (2006).

2. Marques, S. et al. Transcriptional convergence of oligodendrocyte lineage progenitors during development. Dev. Cell 46, 504-517.e7 (2018).

3. Minocha, S. et al. NG2 glia are required for vessel network formation during embryonic development. eLife 4, pii: e09102 (2015).

4. Hill, R. A., Patel, K. D., Goncalves, C. M., Grutzendler, J. \& Nishiyama, A. Modulation of oligodendrocyte generation during a critical temporal window after NG2 cell division. Nat. Neurosci. 17, 1518-1527 (2014). 
5. Hughes, E. G., Kang, S. H., Fukaya, M. \& Bergles, D. E. Oligodendrocyte progenitors balance growth with self-repulsion to achieve homeostasis in the adult brain. Nat. Neurosci. 16, 668-676 (2013).

6. Wamsley, B. \& Fishell, G. Genetic and activity-dependent mechanisms underlying interneuron diversity. Nat. Rev. Neurosci. 18, 299-309 (2017).

7. Gelman, D. et al. A wide diversity of cortical GABAergic interneurons derives from the embryonic preoptic area. J. Neurosci. 31, 16570-16580 (2011).

8. Gelman, D. M. et al. The embryonic preoptic area is a novel source of cortical GABAergic interneurons. J. Neurosci. 29, 9380-9389 (2009).

9. Voronova, A. et al. Migrating interneurons secrete fractalkine to promote oligodendrocyte formation in the developing mammalian brain. Neuron $\mathbf{9 4}$ 500-516.e9 (2017).

10. Winkler, C. C. et al. The dorsal wave of neocortical oligodendrogenesis begins embryonically and requires multiple sources of sonic hedgehog. J. Neurosci. 38, 5237-5250 (2018).

11. Lin, S. C. \& Bergles, D. E. Synaptic signaling between GABAergic interneurons and oligodendrocyte precursor cells in the hippocampus. Nat. Neurosci. 7, 24-32 (2004).

12. Vélez-Fort, M., Maldonado, P. P., Butt, A. M., Audinat, E. \& Angulo, M. C. Postnatal switch from synaptic to extrasynaptic transmission between interneurons and NG2 cells. J. Neurosci. 30, 6921-6929 (2010).

13. Zonouzi, M. et al. GABAergic regulation of cerebellar NG2 cell development is altered in perinatal white matter injury. Nat. Neurosci. 18, 674-682 (2015).

14. Balia, M. et al. Postnatal down-regulation of the GABAA receptor gamma2 subunit in neocortical NG2 cells accompanies synaptic-toextrasynaptic switch in the GABAergic transmission mode. Cereb. Cortex 25, 1114-1123 (2015).

15. Orduz, D. et al. Interneurons and oligodendrocyte progenitors form a structured synaptic network in the developing neocortex. eLife 4, e06953 (2015).

16. Southwell, D. G. et al. Intrinsically determined cell death of developing cortical interneurons. Nature 491, 109-113 (2012).

17. Teissier, A. et al. A novel transient glutamatergic population migrating from the pallial-subpallial boundary contributes to neocortical development. J. Neurosci. 30, 10563-10574 (2010).

18. Bielle, F. et al. Multiple origins of Cajal-Retzius cells at the borders of the developing pallium. Nat. Neurosci. 8, 1002-1012 (2005).

19. Ziskin, J. L., Nishiyama, A., Rubio, M., Fukaya, M. \& Bergles, D. E. Vesicular release of glutamate from unmyelinated axons in white matter. Nat. Neurosci. 10, 321-330 (2007).

20. Suzuki, R. \& Shimodaira, H. Pvclust: an R package for assessing the uncertainty in hierarchical clustering. Bioinformatics 22, 1540-1542 (2006).

21. R Core Team.R: A Language and Environment for Statistical Computing. $\mathrm{R}$ Foundation for Statistical Computing (R Development Core Team, Vienna, Austria, 2014).

22. Hirrlinger, P. G. et al. Expression of reef coral fluorescent proteins in the central nervous system of transgenic mice. Mol. Cell Neurosci. 30, 291-303 (2005).

23. Balia, M., Benamer, N. \& Angulo, M. C. A specific GABAergic synapse onto oligodendrocyte precursors does not regulate cortical oligodendrogenesis. Glia 65, 1821-1832 (2017).

24. Micheva, K. D. et al. A large fraction of neocortical myelin ensheathes axons of local inhibitory neurons. Elife 5, e15784 (2016).

25. Stedehouder, J. et al. Fast-spiking parvalbumin interneurons are frequently myelinated in the cerebral cortex of mice and humans. Cereb. Cortex 27, 5001-5013 (2017).

26. Sun, L. O. et al. Spatiotemporal control of CNS myelination by oligodendrocyte programmed cell death through the TFEB-PUMA axis. Cell 175, 1811-1826.e21 (2018)

27. Cruikshank, S. J., Lewis, T. J. \& Connors, B. W. Synaptic basis for intense thalamocortical activation of feedforward inhibitory cells in neocortex. Nat. Neurosci. 10, 462-468 (2007).

28. Causeret, F., Coppola, E. \& Pierani, A. Cortical developmental death: selected to survive or fated to die. Curr. Opin. Neurobiol. 53, 35-42 (2018).

29. Wong, F. K. et al. Pyramidal cell regulation of interneuron survival sculpts cortical networks. Nature 557, 668-673 (2018).

30. Yu, Y.-C., Bultje, R. S., Wang, X. \& Shi, S.-H. Specific synapses develop preferentially among sister excitatory neurons in the neocortex. Nature 458, 501 (2009).

31. Brown, K. N. et al. Clonal production and organization of inhibitory interneurons in the neocortex. Science 334, 480-486 (2011).

32. Ciceri, G. et al. Lineage-specific laminar organization of cortical GABAergic interneurons. Nat. Neurosci. 16, 1199-1210 (2013).

33. Harwell, C. C. et al. Wide dispersion and diversity of clonally related inhibitory interneurons. Neuron 87, 999-1007 (2015).

34. Mayer, C. et al. Clonally related forebrain interneurons disperse broadly across both functional areas and structural boundaries. Neuron 87, 989-998 (2015).

35. Zhang, X.-J. et al. Precise inhibitory microcircuit assembly of developmentally related neocortical interneurons in clusters. Nat. Commun. 8, 16091 (2017).
36. Butt, S. J. B. et al. The requirement of Nkx2-1 in the temporal specification of cortical interneuron subtypes. Neuron 59, 722-732 (2008).

37. Marques, S. et al. Oligodendrocyte heterogeneity in the mouse juvenile and adult central nervous system. Science 352, 1326-1329 (2016).

38. Fino, E. \& Yuste, R. Dense inhibitory connectivity in neocortex. Neuron 69, 1188-1203 (2011)

39. Crawford, A. H., Tripathi, R. B., Richardson, W. D. \& Franklin, R. J. M. Developmental origin of oligodendrocyte lineage cells determines response to demyelination and susceptibility to age-associated functional decline. Cell Rep. 15, 761-773 (2016).

40. Raff, M. C. et al. Programmed cell death and the control of cell survival: lessons from the nervous system. Science 262, 695-700 (1993).

41. Trapp, B. D., Nishiyama, A., Cheng, D. \& Macklin, W. Differentiation and death of premyelinating oligodendrocytes in developing rodent brain. J. Cell Biol. 137, 459-468 (1997)

42. Franklin, R. J. M. \& Ffrench-Constant, C. Regenerating CNS myelin - from mechanisms to experimental medicines. Nat. Rev. Neurosci. 18, 753-769 (2017)

43. Takeuchi, O. et al. Essential role of BAX,BAK in B cell homeostasis and prevention of autoimmune disease. Proc. Natl Acad. Sci. USA 102, 11272-11277 (2005).

44. Rothman, J. S. \& Silver, R. A. NeuroMatic: an integrated open-source software toolkit for acquisition, analysis and simulation of electrophysiological data. Front. Neuroinform. 12, 14 (2018).

\section{Acknowledgements}

This work was supported by grants from Fondation pour la Recherche Médicale (FRM, «Equipe FRM DEQ20150331681»), Agence Nationale de la Recherche (ANR, ANR-14 CE13-0023), Fondation pour l'aide à la recherche sur la Sclérose en Plaques (ARSEP), ANR under the frame of Neuron Cofund (Era-Net Neuron; project No. R19068KK) and a subaward agreement from the University of Connecticut with funds provided by Grant No. RG-1612-26501 from National Multiple Sclerosis Society (NMSS) (to M.C.A.), from IDEX-Paris Cité Sorbonne (TransGABA) (to M.C.A. and A.P.) and from FRM («Equipe FRM DEQ20130326521») (to A.P.). D. Ort. is recipient of an INSPIRE PhD fellowship from Marie Skłodowska-Curie Action (European Union's Horizon 2020) and Université de Paris (grant agreement: 665850). M.C.A. and A.P. are CNRS (Centre National de la Recherche Scientifique) investigators and teams of the ENP-Ile-de-France network.

\section{Author contributions}

D.Ord., N.B. and D.Ort. conducted electrophysiological experiments, immunostainings and analysis of data. L.V. helped with animal maintenance. M.C.A. performed hierarchical cluster analysis. D.Ord., N.B., D.Ort., E.C., A.P. and M.C.A. designed experiments, and A.P. and M.C.A. supervised the project. D.Ord., N.B., D.Ort., E.C., A.P. and M.C.A. wrote the manuscript.

\section{Additional information}

Supplementary Information accompanies this paper at https://doi.org/10.1038/s41467 019-11904-4.

Competing interests: The authors declare no competing interests.

Reprints and permission information is available online at http://npg.nature.com/ reprintsandpermissions/

Peer review information Nature Communications thanks Akiko Nishiyama, and the other, anonymous, reviewer(s) for their contribution to the peer review of this work. Peer reviewer reports are available.

Publisher's note Springer Nature remains neutral with regard to jurisdictional claims in published maps and institutional affiliations.

Open Access This article is licensed under a Creative Commons Attribution 4.0 International License, which permits use, sharing, adaptation, distribution and reproduction in any medium or format, as long as you give appropriate credit to the original author(s) and the source, provide a link to the Creative Commons license, and indicate if changes were made. The images or other third party material in this article are included in the article's Creative Commons license, unless indicated otherwise in a credit line to the material. If material is not included in the article's Creative Commons license and your intended use is not permitted by statutory regulation or exceeds the permitted use, you will need to obtain permission directly from the copyright holder. To view a copy of this license, visit http://creativecommons.org/ licenses/by/4.0/

(C) The Author(s) 2019 